A N N A L E S Annales de Bretagne et des Pays de l'Ouest

Anjou. Maine. Poitou-Charente. Touraine

115-1 | 2008

Varia

\title{
Les deux bœufs du déluge et la submersion de la ville d'Is
}

\section{Claude Sterckx}

\section{(2) OpenEdition}

1 Journals

Édition électronique

URL : http://journals.openedition.org/abpo/352

DOI : $10.4000 /$ abpo.352

ISBN : 978-2-7535-1509-3

ISSN : 2108-6443

\section{Éditeur}

Presses universitaires de Rennes

Édition imprimée

Date de publication : 30 mars 2008

Pagination : 15-53

ISBN : 978-2-7535-0653-4

ISSN : 0399-0826

\section{Référence électronique}

Claude Sterckx, "Les deux bœufs du déluge et la submersion de la ville d'Is », Annales de Bretagne et des Pays de l'Ouest [En ligne], 115-1 | 2008, mis en ligne le 30 mars 2010, consulté le 19 avril 2019. URL : http://journals.openedition.org/abpo/352 ; DOI : 10.4000/abpo.352 


\title{
Les deux bœufs du déluge et la submersion de la ville d'Is
}

\author{
Claude STERCKX \\ Maître d'enseignement à l'université Libre de Bruxelles \\ Professeur à la Faculté ouverte des religions \\ et des humanismes laïques de Charleroi
}

Le recoupement de traditions disparates du Pays de Galles permet de reconstituer un récit mettant en scène deux rois légendaires métamorphosés en bœufs. Les thèmes mis en œuvre associent ceux-ci à une formation originelle du pays, puis à un risque d'ennoiement eschatologique de celuici. Ầ partir de cette reconstitution, il est possible de repérer des éléments de ce même thème dans les autres traditions celtes, jusqu'à la fameuse légende armoricaine de la ville d'Is. Ces rapprochements permettent, selon toute vraisemblance, de soupçonner derrière tous ces récits un écho de très anciennes croyances, sans doute préchrétiennes. Commençons par ouvrir le dossier gallois relatif à Nynnio et Peibio.

\section{Deux bœufs}

Les frères Nynnio et Peibio sont deux rois pseudo-historiques ${ }^{1}$ du Pays de Galles le plus ancien. Leur mère, anonyme, est dite fille d'un roi Custennin dont la personnalité n'est pas précisée ${ }^{2}$ mais les associations récurrentes de Nynnio et de Peibio avec le matériel arthurien laissent supposer qu'il n'est pas impossible qu'il s'agisse de Custennin Bendigaid, le grand-père paternel d'Arthur ${ }^{3}$. Leur père est un obscur Erb ab Erbic, que deux chartes du sixième siècle qualifient de roi de Gwent et d'Ergyng ${ }^{4}$. Quant à leur histoire, elle se laisse reconstruire comme suit.

1. Du moins dans les légendes qui leur sont attachées.

2. Charte de donation de Bicknor on the Wye à saint Dyfrig in RHŶs, J., Evans, J. G., The Text of the Book of Llan Dâv, Oxford, 1893, p. 72.

3. L'hypothèse a déjà été émise par S. Baring-Gould et J. Fisher, The Lives of the British Saints, Londres, 1907-1913, II, p. 177 et 375. Voir le tableau généalogique proposé plus loin.

4. P. C. Bartrum, A Welsh Classical Dictionary, Aberystwyth, 1993, p. 253. 
Après la mort de leur père, ils se partagent son héritage de sorte que Nynnio devient roi de Gwent ${ }^{5}$ et Peibio roi d'Ergyng ${ }^{6}$ mais une folle querelle en vient à les opposer : Peibio prétend que les étoiles forment un troupeau de moutons qui lui appartient, Nynnio que le ciel est une pâture dont il est propriétaire et interdit que les " moutons " de son frère s'y repaissent! Leur dispute leur fait alors se mener une telle guerre qu'ils se trouvent hors d'état de résister lorsqu'un géant, Rhita, les attaque : ils sont vaincus et Rhita leur coupe leurs deux barbes en guise de trophées. Vingt-huit autres rois de Grande-Bretagne se liguent aussitôt contre le géant mais ils sont tous vaincus et Rhita ajoute leurs vingt-huit barbes à celles de Nynnio et de Peibio pour s'en faire un manteau?

Rhita ne triomphe pas longtemps. Il ose en effet défier Arthur lui-même, auquel il enjoint soit de lui envoyer sa barbe pour qu'elle soit ajoutée - à une place d'honneur - à son manteau, soit de se mesurer en duel avec lui. Le duel est livré au sommet du Snowdon et c'est, bien sûr, Arthur qui tue le géant et lui prend sa barbe et son manteau ${ }^{8}$.

Sans qu'on en connaisse précisément la cause, le roi Peibio se trouve aussi affecté par une peu ragoûtante infirmité : il bave sans arrêt et en telle abondance que deux serviteurs doivent sans cesse lui éponger la bouche, de sorte qu'il en garde le surnom de Peibio Glaforog " le Baveux " :

"In Herefordshire in a parish church is the picture of a king with a man on each side of him, with napkins wiping the rheum and drivel from his mouth, that humour so abounding in him that he could get no cure of it, which king the country people call King Dravellor, the Britains Pepian Glanorawc, the Latins Pepianus Spumosus rex Ereichy, i.e. king of Urchenfield ${ }^{9}$."

Dans cet état, il est le triste héros d'une légende qui mérite d'être rapportée en détail. Découvrant que sa fille Erfddyl est enceinte alors qu'elle n'est pas encore mariée, Peibio la condamne à mort. Il la fait d'abord coudre dans un sac et jeter à l'eau mais, à chaque fois qu'elle est ainsi jetée, la rivière la ramène saine et sauve sur la berge. Peibio la fait alors brûler vive... et on la retrouve le lendemain, bien vivante, avec son fils nouveau-né sur son sein. Peibio, cette fois, se laisse attendrir : il embrasse l'enfançon et

5. Ibidem, p. 511

6. Vita sancti Dubricii 1 in J. Rhŷs, J. G. Evans, op. cit., p. 78; etc.

7. T. Williams, The Iolo Manuscripts, Abergavenny, 1848, p. 193-194 (où E. Williams, le fameux " Iolo Morganwg " prétend avoir trouvé cette histoire dans un Llyfr Iaco ab Dewi inconnu par ailleurs)

8. Geoffrey de Monmouth, Historia regum Britanniae 165 in N. Wright, The Historia regum Britanniae of Geoffrey of Monmouth. I. Bern, Burgerbibliothek Ms. 568, Cambridge, 1985, p. 119. Une autre version, collectée au début du seizième siècle par Siôn Dafydd Rhŷs d'après des traditions orales, situe le duel près de Mawddwy, dans la même région, et précise que, avant la victoire d'Arthur, les deux duellistes se sont arrachés mutuellement la barbe, d'où le nom de Rhiw y Barfau "le Mont des Barbes " donné à la colline sur les flancs de laquelle s'est déroulée l'empoignade (in H. Owen, "Peniarth Ms. 118, fo. 829-837 ", Y Cymmrodor XXVIII, 1917, p. 126-129).

9. J. Lewis, The History of Great Britain, Londres, 1729, p. 43. 
s'en trouve miraculeusement guéri de son infirmité baveuse car le garçonnet n'est autre que Dyfrig (Dubricius), qui deviendra l'un des plus grands saints gallois ${ }^{10}$.

La très catholique Vita sancti Dubricii ici résumée, ne révèle pudiquement pas le nom du séducteur d'Erfddyl car il s'agit de son propre père, ainsi que le révèle une glose marginale :

"The above named king of Erching, named Pepiau, was father of st. Dubricius as is held in the chronicles at the college of Warwick and above the name of the said king "father of st. Dubricius " was formerly written correctly in an antique hand, and some later [person] wished to change it as above but mutilated the antique writing and spoilt it ${ }^{11}$."

De même, au dix-septième siècle, John Usher note encore :

"It is not known who [Dubricius'] father really was. Some bunglers therefore falsely declare him to be without a father [...]. Another [that] he was a king of Erchyng named Pepiau ${ }^{12}$."

Les crimes de Nynnio et de Peibio les font finalement condamner à être métamorphosés en bœufs de labour, ainsi qu'un autre géant, Ysbaddaden Pencawr, l'explique à Culhwch, un neveu d'Arthur auquel il essaie de ne pas donner la main de sa fille en lui imposant mille tâches surhumaines, culminant dans la traque du monstrueux sanglier Trwyth et de sa harde :

"Quand j'aurai obtenu tout ce que je vais te demander, tu auras ma fille [...]. Il te faudra les deux bœufs Gwinau [Châtain] et Gwlwlwyd [Gris Pommelé], équipiers d'attelage [...]. Je veux aussi Melyn Gwanwyn [Jaune de Printemps] et Ych Brych [Bœuf Pie] dans ce même attelage [...]. Les deux Ychen Bannog [Bœufs Cornus], dont l'un est de l'autre côté des monts Bannock ${ }^{13}$ et le second de ce côté-ci, il faudra les atteler ensemble à la même charrue : ceux-là sont Nynnio et Peibio, que Dieu a transformés en bœufs à cause de leurs péchés ${ }^{14}$."

Remarquablement, une autre tâche prétendument impossible imposée à Culhwch est d'acquérir

10. Vita sancti Dubricii 1-2 in J. Rhŷs , J. G. Evans, op. cit, p. 78-79.

11. Ibidem, p. 337.

12. J. Usher, Britannicarum ecclesiarum antiquitates, Londres, 1687, p. 238.

13. Les monts Bannock, entre Stirling et Dumbarton, dans l'actuel Strathclyde, formaient frontière entre la Pictavie et la Cumbrie dans les plus anciennes traditions cambriennes (R. Bromwich, D. S. Evans, Culhwch and Olwen, Cardiff, 1992, p. 123-124.)

14. Mal y cafas Culhwch Olwen in R. Bromwich, D. S. Evans, op. cit., p. 21-22. Cf. P.Y. Lambert, , Les Quatre Branches du Mabinogi et autres contes gallois du Moyen Âge, Paris, 1993, p. 143-144 (dont, ici comme plus loin, nous suivons à peu près la traduction). Les deux premières paires de bœufs, formant évidemment triade avec le couple Nynnio - Peibio, se retrouvent déjà dans une triade plus ancienne : "Trois bœufs fameux de Grande-Bretagne : Melyn Gwanwyn, Gwinau le bœuf gris pommelé et Ych Brych " (Trioedd Ynys Prydain 45 in R. Bromwich, Trioedd Ynys Prydain, Cardiff, 2006², p. 124), dans laquelle la ligne Gvineu ych gwylwylyd peut aussi se comprendre "Gwinau, le bœuf de Gwlwlwyd ", d'où sans doute le dédoublement qui apparaît dans Mal y cafas Culhch Olwen. 
" une laisse faite de la barbe de Dillus Barfog "le Barbu" [...] et on ne pourra l'utiliser que si on lui arrache les poils de sa barbe de son vivant et si on les arrache avec une pince en bois. Il ne laissera personne lui faire cela de son vivant mais la barbe ne servira à rien s'il est mort car elle sera cassante $^{15} "$.

Cette fois, ce sont Cai et Bedwyr qui rencontrent le géant, et c'est au sommet du Plynlimon qu'ils lui prennent sa barbe avant de le tuer ${ }^{16}$ mais il n'y a pas de doute qu'il y a là une simple variante de l'histoire du géant Rhita ${ }^{17}$.

Pour ce qui est de Nynnio et Peibio, de nombreuses traditions orales content la fin de leur histoire, après qu'ils ont été réunis (puisque Culhwch, aidé par Arthur et ses hommes, mène à bien toutes les tâches imposées par Ysbaddaden Pencawr). L'une d'elle conte qu'un monstre aquatique hante les eaux du Llyn yr Afanc "le Lac du Monstre", près de Betws y Coed (Gwynedd). Une vierge réussit à l'attirer en lui présentant son sein nu mais la bête le lui arrache d'un coup de griffe et regagne son antre. Ce sont alors les deux Ychen Bannog, Nynnio et Peibio, qui réussissent à l'en tirer, non sans que l'un d'eux ${ }^{18}$ perde un oil dans l'affaire, si grand qu'il constitue encore aujourd'hui le petit lac dit Llygad Ych " l'Eil de Bouf ${ }^{19}$ ".

Plus significativement, Nynnio et Peibio réussissent similairement à tirer, cette fois hors du Llyn Lliwan, un autre monstre aquatique responsable, lui, d'une inondation de toute la Grande-Bretagne - autrement dit d'une sorte de déluge microcosmique - causé par une éruption de ce lac ${ }^{20}$. Ils sont associés là à la figure de Hu Cadarn, fantôme de la tradition galloise ${ }^{21}$ issu en fait de la version indigène du Pèlerinage de Charlemagne où son nom traduit en fait celui de Hugon le Fort, empereur légendaire de Constantinople ${ }^{22}$, mais il ne fait aucun doute que c'est la coutume prêtée à ce dernier de labourer avec une araire en or qui en a fait le laboureur paradigmatique, seul capable de conduire l'attelage des deux Ychen Bannog.

Enfin, le dernier exploit prêté à cet attelage est d'avoir contribué à l'érection de l'église de Llandewibrefi (Dyfed). Ils y amènent notamment une pierre si lourde qu'elle laisse une trouée à travers la montagne, trouée encore appelée aujourd'hui Cwys yr Ychen Bannog « le Sillon des Ychen

15. Mal y Cafas Culhwch Olwen in Bromwich, R., Evans, D. S., op. cit., p. 26.

16. Mal y cafas Culhwch Olwen, ibidem, p. 34-35.

17. R. Bromwich, D. S. Evans, Culhwch ac Olwen, Cardiff, 1988, p. LVI-LVIII.

18. Peut-être Peibio car il est réputé avoir été guéri d'un mal de la vue par saint David : R. Bromwich, D. S. Evans, op. cit., p. 124-125.

19. J. Rhŷs, Celtic Folk-Lore, Oxford, 1901, p. 130-132. Sur le sens de la métaphore, cf. C. Sterckx, « L'œil, la main, le pied : ordalies trifonctionnelles », 2007, (à paraître).

20. J. Rhŷs, op. cit., p. 142.

21. Tardivement transformé en héros majeur dans les délires néo-druidiques de Iolo Morganwg.

22. R. Bromwich, "Trioedd Ynys Prydain: The Myvyrian 'Third Series' ". Transactions of the Honourable Society of Cymmrodorion, 1968-1969, p. 323; A. C. Rejhon, " Hu Gadarn : Folklore and Fabrication ", in P. K. Ford (ed.), Celtic Folklore and Christianity. Studies in Memory of William H. Heist, Santa Barbara, 1983, p. 201-212. 
Bannog ". Cette fois pourtant, ils succombent à la tâche, non sans que l'un d'eut n'ait mugi neuf fois si fort qu'il en fendît le dernier obstacle qui s'opposait à eux ${ }^{23}$. Ne resterait d'eux qu'une de leurs cornes : le Mabcorn yr Ychen Bannog (" Os de Corne des Ychen Bannong "), en fait un os de corne d'urus longtemps conservé comme relique dans l'église de Llandewibrefi, désormais conservé dans les vitrines du Welsh Folk Museum à Saint Fagans ${ }^{24}$.

Cette reconstitution - nous le reconnaissons et insistons même sur cette reconnaissance - est artificielle. Non seulement elle n'est que la mise bout à bout, purement arbitraire, de traditions disparates qu'aucun document ne rassemble ni n'associe mais, en outre, certaines de ces traditions - tout particulièrement celles dues à Iolo Morgannwg - sont extrêmement suspectes. Notre reconstitution n'a pour but que d'exposer commodément le matériel. Ce ne sont, heureusement, ni la séquence des épisodes ni les détails les plus suspects qui nous intéressent ici.

\section{Le sein coupé}

Bien qu'issus de traditions orales, un certain nombre de motifs trouvent, néanmoins, des comparaisons remarquables. Le sein que le monstre arrache à la vierge qui veut lui faire quitter son repaire aquatique pour sauver le pays -autrement dit le monde - renvoie ainsi à un motif bien attesté dans les traditions celtes et dérivées, mais également présent dans d'autres traditions indo-européennes, ce qui assure vraisemblablement l'antiquité de son origine.

Le parallèle le plus évident se trouve dans la légende de Caradoc le Jeune $^{25}$, telle que préservée par un continuateur anonyme de Chrétien de Troyes. Celui-là narre comment le roi Caradoc l'Ancien épouse la princesse Isaune et comment aussi l'enchanteur Eliavrès, amant de cette dernière, le berne en substituant magiquement à la reine successivement une truie, une jument et une lice. Ainsi, lorsque Isaune accouche d'un fils, Caradoc le Jeune, le vrai père est Eliavrès et non le roi.

L'enfant Caradoc est présenté en page à la Cour d' Arthur. Il y apprend le secret de sa naissance et dénonce aussitôt la forfaiture à son père putatif : celui-là fait emprisonner Isaune et condamne Eliavrès à engrosser successivement les mêmes bêtes qu'il avait susbtituées à la reine dans le lit royal $^{26}$. Eliavrès et Isaune complotent ensuite pour se venger du fils qui les a trahis. Isaune demande au jeune Caradoc d'ouvrir une armoire dans laquelle ils ont caché un serpent monstrueux. Dès qu'il est libéré, celui-là

23. J. Rhŷs, op. cit., p. 576-579,

24. R. Gwyndaf, Chwedlau gwerin Cymru, Cardiff, 1992, p. 68.

25. Autrement dit le Caradog Breichfras gallois, fils de Llŷr, le dieu Océan de la mythologie celte, et époux de Tegau Eurfron "Au Sein d'Or ", ce qui garantit que le conte résumé ici est effectivement d'origine galloise : cf. P. C. Bartrum, A Welsh Classical Dictionary, op. cit., p. 102-104.

26. Il y a là un motif mythologique indo-européen majeur : voir C. Sterckx, Le fils parfait et ses frères animaux, Bruxelles, 2002. 
s'attache au bras du jeune prince et, ainsi lové, il lui suce la vie, le vouant au trépas dans les deux ans ou à la mort immédiate si on le blesse dans cette position. Heureusement, Cador, un ami du jeune prince, réussit à extorquer à Isaune le secret de sa guérison : Caradoc devra se tenir dans un cuveau de vinaigre et une vierge, nue au moins jusqu'à la ceinture, devra tendre son sein au serpent. La sœur de Cador se dévoue : le monstre lâche le bras pour sa gorge... mais un coup d'épée tranche le sein de la courageuse fille et le reptile peut enfin être détruit. Caradoc le Jeune épouse, bien sûr, celle qui l'a sauvé. Plus tard, il conquiert une boucle ${ }^{27}$ en or ayant le pouvoir de guérir toute mutilation : il la donne à son épouse, pour laquelle la boucle se transforme en sein d'or, lui rendant ainsi son intégrité physique ${ }^{28}$.

On reconnaît évidemment que, dans les deux contes, le monstre fatal est semblablement attiré hors d'un milieu liquide - lac ou cuveau de vinaigre - par une vierge, au prix de l'un de ses seins.

\section{Le manteau de barbes}

L'histoire du manteau de barbes, qui fait florès par la suite dans toute la littérature arthurienne ${ }^{29}$, a pour sa part un parallèle éventuel dans la tradition ossète selon laquelle le héros Soslan se fait faire, lui,

" une pelisse avec des peaux humaines : peaux de crânes et peaux de lèvres supérieures avec la moustache ${ }^{30}$. Il ne parla de la chose à personne mais se mit à tuer des hommes et, leur écorchant le crâne et la lèvre supérieure, réunit ce qu'il fallait pour la pelisse [...]. Il y avait quelque part trois jeunes filles : c'est à elles que Soslan porta les peaux. Quand elles les regardèrent, elles reconnurent chacune la tête d'un oncle, d'un frère, et furent dans un grand embarras [...].

"Taillez-lui une pelisse, leur dit Syrdon, et cousez-la, seulement laissez un vide par devant ${ }^{31}$. Quand il viendra, mettez-la sur lui et dites-lui : 'Ta pelisse te va bien mais il lui manque de quoi faire le revers.' Si tu nous apportes la peau du crâne d'Eltagan, le fils de Kutsykk - on dit qu'elle est en or -, nous pourrons l'achever. Mais il faut cette peau-là : aucune autre ne ferait l'affaire..." [...].

27. C'est le terme technique qui désigne la bosse au centre d'un bouclier (d'où d'ailleurs le nom de ce dernier), protégeant la main qui le tient.

28. Livre de Carados in W. Roach et al., The Continuations of the Old French Perceval of Chrétien de Troyes, Philadelphie, 1949-1983, I, p. 84-238, II, p. 195-377, III 1 p. 131-204. Cf. G. Paris, " Caradoc et le serpent ", Romania, XXVIII, 1899, p. 214-231; R.S. Loomis, " L'étrange histoire de Caradoc de Vannes ", Annales de Bretagne, LXX, 1963, p. 165-175. Pour les importants parallèles celtes et indo-européens, ainsi que pour l'exégèse du mythème : G. Le Menn, La femme au sein d'or, Saint-Brieuc, 1986; C. Sterckx, La mutilation rituelle des ennemis et les concepts de l'âme chez les Celtes préchrétiens, Paris, 2005, p. 127-133.

29. J. D. Bruce, The Evolution of Arthurian Romance, Baltimore, 1928, II p. 319-320; K. Hodder et al., Dynastic Romance, in W. R. J. Barron, The Arthur of the English, Cardiff, 2001², p. 74, 299 n. 1. Cf. H. Nickel, « The Fight About King Arthur's Beard and for the Cloak of Kings' Beards ", Interpretation XVI, 1985, p. 1-7.

30. Substitut évident des barbes, mode pileuse oblige.

31. C'est la place d'honneur, celle-là même que Rhita réserve pour la barbe d'Arthur. 
[Soslan] le scalpa, rapporta la peau aux jeunes filles qui durent faire le revers de sa pelisse ${ }^{32}$."

Il a été bien relevé que ce conte n'est pas de pure fantaisie mais qu'il conserve la trace d'un trait déjà noté par Hérodote parmi les Scythes, lointains ancêtres des Ossètes :

« De tous ceux qu'il tue à la guerre, le Scythe apporte la tête au roi [...]. Voici comment il écorche cette tête : il fait une incision autour des oreilles, prend un morceau de la peau et la sépare du crâne en la secouant. Il racle ensuite l'intérieur avec une côte de bœuf [...]. Beaucoup aussi, en cousant ensemble plusieurs de ces peaux arrachées, se font des manteaux, à la manière des capes de berger ${ }^{33}$."

\section{Díl}

Latinisé par une désinence en -us, Dillus Barfog se laisse vraisemblablement reconnaître comme au moins l'homonyme de personnages irlandais appelés Díl, qui se retrouvent significativement ci après.

Le premier est le druide borgne Díl mac Dáchreaga qui intervient notamment dans la légende de Corc Duibhne.

Un roi de Munster, Conaire mac Moghalámha, épouse Sáraid, fille du haut-roi d'Irlande Conn Céadchathach qui le désigne alors comme son successeur. Sáraid et Conaire ont trois fils : Aonghus, Eochu et Ailill, appelés aussi les trois Cairbre. Aonghus est Cairbre Músc, Eochu est Cairbre Rioghfhota et Ailill est Cairbre Báscháin.

Après huit ans de règne, Conaire est tué par Neimeadh mac Sraibhghinn qui épouse à son tour Sáraid. Les trois Cairbre vont demander de l'aide à leur oncle maternel Art mac Cuinn et, avec son appui, le vainquent à la bataille de Ceann Abhradh. Neimeadh se réfugie dans le giron de Sáraid : Cairbre Músc l'y poursuit et l'y tue. Plus tard, il achève de mériter son surnom de Músc « Révulsant " en violant sa propre sœur :

\footnotetext{
"Cairbre Músc mac Conaire : c'est lui qui engendra [Corc Duibhne] avec sa sœur Duibhhionn, fille de Conaire, quand il fut roi de Munster. Les récoltes du Munster furent mauvaises à cause de cela.

"Les crimes du roi en sont la cause", avoua Cairbre [à son druide]. "J'ai commis un inceste. Quel en sera le résultat?"

"Deux fils", répondit le druide, "Corc et Cormac".

Ils naquirent jumeaux [...]. Les Munstériens se dirent : "Brûlons-les afin que le pays ne soit pas déshonoré."
}

32. Nous reproduisons la traduction de G. Dumézil, Le livre des héros, Paris, 1965, p. 8183. Il y a, bien sûr, inversion : d'un côté un géant veut se faire un manteau mais est tué par le héros dont la barbe lui manque pour l'achever, de l'autre c'est le héros qui veut se faire une pelisse et qui tue le personnage surhumain dont la moustache lui manque pour l'achever.

33. Hérodote, Historíai LIV in P.-E. Legrand, Hérodote, Paris, 1932-1954, IV, p. 85. Cf. G. Dumézil, Romans de Scythie et d'alentour, 1978, Paris p. 253-255 dont nous suivons ici la traduction. 
"Donnez-moi Corc", dit le druide du palais, "je l'emmènerai hors d'Irlande afin qu'il n'y ait pas de déshonneur ici ${ }^{34}$."

Corc lui fut donné. Le druide et sa cailleach [Baoí] l'emmenèrent sur leur île 35 ..."

Lâge venu, Baoí ramène Corc à sa mère et il est alors placé comme page chez Aonghus Gaíbhuaitheach mais ce dernier éborgne accidentellement le haut-roi, ce qui le contraint à l'exil ainsi que tout son clan. En Leinster, ils adoptent la petite Eithne Uathach ${ }^{36}$ qu'ils nourrissent de la chair de petits enfants, si bien qu'elle est très vite nubile et peut alors épouser Aonghus Gaibhuaitheach. Ils sont toutefois attaqués de tous côtés "comme le sont des sangliers assaillis de toutes parts par des chiens ". L'un de leurs ennemis est précisément Díl mac Dáchreaga, qui cache une baguette magique dans sa chaussure mais dont la propre fille se laisse convaincre par Eithne Uathach de la lui voler et de la donner pour être détruite, ce qui entraîne peu après la mort du druide ${ }^{37}$.

Díl Dáchreage intervient encore dans une autre légende : celle de la bataille de Magh Mucraimhe. Elle conte comment un prince munstérien, Lughaidh Laíghde, engendre avec son épouse Sadhbh un fils appelé Lughaidh mac Con, puis comment Sadhbh, devenue veuve, se remarie avec le roi de Munster Ailill Ólom, qui prend alors en page à sa Cour le jeune Lughaidh mac Con.

Un jour, Lughaidh mac Con et Eoghan Mór, un fils du roi Ailill, s'emparent de Ferfhí, le gardien de la source cosmique ${ }^{38}$. Celui-là n'est pas long à leur échapper mais les deux garçons ne s'en disputent pas moins l'honneur de sa capture. Une bataille s'ensuit, au terme de laquelle Lughaidh, surclassé, doit s'exiler. Il finit par obtenir l'appui du roi de Calédonie pour envahir le Munster et prendre sa revanche sur Eoghan Mór. Les deux armées se renontrent à Magh Mucraimhe.

34. Détail important : dans sa version de l'histoire, Geoffrey Keating, Foras feasa ar Éirin I 44 in D. Comyn, P. S. Dinneen, The History of Ireland by Geoffrey Keating, 1902-1914, Dublin, II, p. 314 précise que Cormac, le jumeau de Corc, est brûlé et que ses cendres sont jetées à l'eau. Cf. P. C. Power, Sex and Marriage in Ancient Ireland, Dublin, 1976, p.17.

35. L'île est Teach Duinn, autrement dit l'Autre Monde (C. Sterckx, « Lugus, Lugh, Lleu... : recherche en paternité ", Ollodagos, X, 1997, p. 46 n. 81). Une cailleach est une vieille répugnante et il s'agit ici de la Cailleach Bhéarra, incarnation de l'Irlande et peut-être ici l'ancêtre mythique de Corca Duibhne que les plus anciennes inscriptions appellent Dovina " la Mauvaise (?)»: F. J. Byrne, Irish Kings and High Kings, Londres, 1973, p. 166-167; D. O hÓgáin, Myth, Legend and Romance, 1990, Londres, p. 67-69, "The River Boyne and the Ancient Seers ", Studia Celtica Japonica, VI, 20. 13-35. Sur Dovina : J. Uhlich, « Dov(a) and Lenited -b- in Ogam ", Ériu, XL, p. 129-133.

36. Sur cette figure : C. Dagger, "Eithne ", Zeitschrift für Celtische Philologie, XLIII, 1989, p. $97-100$.

37. Tucaid ionnarbha nan nDéise i Mumhain agus oideadh Chormaic 9-12 in V. Hull, « The Later Version of the Expulsion of the Déssi ", Zeitschrift für Celtische Philologie, XXVII, 1958-1959, p. 32-39. Cf. De mhacaibh Chonaire in L. Gwyn, "De maccaib Conaire ", Ériu, VI, 1912, p. 144-153.

38. Cf. C. Sterckx, Les dieux protéens des Celtes et des Indo-Européens, Bruxelles, 1994, p. 116 . 
La veille de l'affrontement, Eoghan se rend auprès de Díl pour le prier de venir jeter un sort funeste sur ses adversaires. Le druide pressent la défaite des Munstériens et leur mort à tous deux mais aussi l'éventuelle fortune de sa propre descendance. Il vient donc à Magh Mucraimhe avec sa fille Moncha et il prescrit à cette dernière de se donner à Eoghan Mór, puis, neuf mois plus tard, de retenir son accouchement pendant une nuit et un jour car la descendance du fils qui naîtra ainsi sera assurée de tenir la souveraineté du Munster à jamais. Moncha obéit, en meurt mais met au monde Fiachu Muilleathan, source de tous les rois munstériens subséquents ${ }^{39}$.

Ajoutons à cela qu'une autre version de la légende signale que le druide Díl s'appelait aussi Triath ${ }^{40}$ et que la plaine de Magh Mucraimhe, où sont situés tous les épisodes qui le concernent, a elle-même une histoire remarquable. quer.

«Magh Mucraimhe : d'où vient ce nom? Ce n'est pas difficile à expli-

Une harde de sangliers druidiques [= surnaturels, métamorphosés] sortit de l'Oweynagat à l'époque d'Ailill et de Meadhbh ${ }^{41}$. Ces sangliers détruisaient le blé et le lait partout où ils passaient et aucun homme n'était capable de les dénombrer exactement ${ }^{42}$. Ailill et Meadhbh vinrent à Fraochmhagh pour leur donner la chasse et ils les traquèrent jusqu'à Bealach na Feat. Là, Meadhbh réussit à en saisir un par la patte mais il s'échappa en lui laissant sa peau dans la main. Après cela, ils purent être dénombrés dans cette plaine et c'est de là que vient son nom de Magh Mucraimhe "Plaine du Dénombrement des Porcs" ${ }^{43}$."

Ailleurs enfin, Triath n'est pas Díl mais l'un de ses très remarquables compagnons :

"Brighid la poétesse, fille du Daghdha [Eochaidh Ollathair] : c'est à elle qu'appartenaient les deux bœufs royaux de Díl, Fea et Feimhean de qui Magh

39. Cath Maighe Mucraimhe in M. O'Daly, Cath Maige Mucrama, Londres, 1975. Cf. Cóir anman 42 in W. Stokes, (1897) Cóir anman, in W. Stokes, E. Windisch, Irische Texte, Leipzig, 1880-1905, III, p. 306-308; Sceala Eoghain agus Chormaic in T. Ó Cathasaigh, The Heroic Biography of Cormac mac Airt, Dublin, 1977. Voir aussi M. Dillon, The Cycle of the Kings, Oxford, 1946, p. 16-23; A. D. Rees, B. Rees, Celtic Heritage, Londres, 1961, p. 220 ; F.J. Byrne, op. cit., p. 291; D. Ó hÓgáin, op. cit., p. 202-203 et 277-279.

40. Fiachu Muilleathan in W. Stokes, "A Note About Fiachu Muillethan ", Revue Celtique, XI, 1890, p. 41-45.

41. L'Oweynagat est une bouche de l'Autre Monde située à proximité de la résidence royale d'Ailill et de Meadhbh, roi et reine du Connaught (cf. N. Stalmans, E. Snow, "L'Oweinagat », Ollodagos, IV, 1992-1993, p. 289-301).

42. Le fait de dénombrer des êtres surnaturels est le moyen traditionnel de les exorciser : K. H. Jackson, A Celtic Miscellany, Harmondsworth, 1971, p. 314 n.143. Le récit du Cath Maighe Mucraimhe 36 in M. O'Daly, op. cit., p. 50 ajoute « il n'était pas possible non plus de les tuer car ils devenaient invisibles dès qu'on levait une arme contre eux ".

43. Dinnsheanchas de Rennes 70 in W. Stokes, "The Prose Tales in the Rennes Dindsenchas ", Revue Celtique, XV, p. 470. Cf. Dinnsheanchas métrique in E. J. Gwynn, The Metrical Dindsenchas, Dublin, 1903-1935, III, p. 382-384; Cath Maighe Mucraimhe 36-37 in M. O'Daly, op. cit., p. 50-51 (qui achève significativement l'histoire en précisant que, après leur dénombrement, les sangliers disparurent et que " nul ne sait où ils allèrent "). 
Fea et Magh Feimhin tirent leurs noms. Il y avait avec eux Triath, le roi des sangliers, de qui Trethirne tire son nom ${ }^{44} . . . "$

Et sa nature de sanglier est bien confirmée par le vénérable Sanas Chormaic:

"Triath a trois sens, soit "roi", "mer" et "sanglier". On les distingue néaoins par leurs génitifs [...]. Triath "roi" : c'est ce qui maintient le pays en paix; triath "mer", c'est ce qui terrifie le pays; triath "sanglier", c'est ce qui ravage le pays ${ }^{45} \ldots$ ".

tout comme par la reconnaissance qu'il est la forme irlandaise d'une figure mythique pancelte encore connue en Galles sous la forme de Trwyth et en Écosse sous celle de Troit.

Car si Troit n'est plus qu'un écho fugitif ${ }^{46}$, les parallèles entre les traditions irlandaises et les traditions galloises sont remarquables.

\section{Défrichements}

La notule topologique associant le sanglier Triath aux deux bœufs royaux, Fea et Feimhean, attribue à ces derniers le défrichage de deux grandes plaines auxquelles ils auraient laissé leurs noms : Magh Fea en Leinster et Magh Feimhin en Munster.

Mais plusieurs versions concurrentes circulaient, dues manifestement à des homonymies ou des paronymies.

L'une d'elles attribue le défrichage de trois plaines - outre Magh Fea et Magh Feimhin, Magh Feara en Munster - à trois frères : Fea, Feara et Feimhean, fils de Mogach, des serfs au service des Milésiens, c'est-à-dire des derniers conquérants mythiques de l'Irlande selon la sociogonie qui lui tient lieu de mythe constitutif ${ }^{47}$.

Une autre ne concède à ces trois que le défrichage de deux plaines et attribue celui de Magh Fea à une déesse Fea, fille de Neachtan Ealcmhar, mais sans expliquer pourquoi elle lui aurait laissé son nom ${ }^{48}$.

44. Leabhar Gabhála Éireann VII 317 in R. A. S. MacAlister, Lebor Gabála Érenn, Dublin, 1938-1956, IV, p. 132. Nous insérons en italiques les détails qu'ajoutent deux autres versions de la notule (VII 314, 344 in R. A. S. MacAlister, op. cit., IV, p. 122, 158).

45. Sanas Chormaic 1202 in K. Meyer, «Sanas Cormaic ", in O. Bergin et al, Anecdota from Irish Manuscripts. Halle, 1913, p. 104. Il y a sans doute ici une formule trifonctionnelle : C. Sterckx, Sangliers Père \& Fils, Bruxelles, 1998, p. 73 n.1.

46. Pour ce dernier : C. Sterckx, Sangliers Père \& Fils, op. cit., p. 75.

47. Fea, Feara et Feimhean sont signalés comme des serfs des Milésiens par une note du Livre de Ballymote in E. J. Gwynn, The Metrical Dindsenchas, op. cit., III, p. 511. Sur les conquêtes mythiques de l'Irlande: A. D. Rees, B. Rees, Celtic Heritage, op. cit., p. 95-117; C. Sterckx, "Débris mythologiques en Basse-Bretagne ", in G. Le Menn, J.-Y. Le Moing (éd.), Bretagne et pays celtiques : Mélanges offerts à la mémoire de Léon Fleuriot, PUR, Rennes, 1992, p. 54-58.

48. Peut-être y a-t-il une allusion dans un vers du Dinnsheanchas métrique qui paraît devoir se comprendre "Magh Fea, qu'une main féminine n'a pas suffi à tenir " : cf. E. J. Gwynn, The Metrical Dindsenchas, op. cit., III, p. 512, n. 15. Sur Fea : J. MacKillop, Dictionary of Celtic Mythology, Oxford, 1998, p. 182. 
Les deux bœufs du déluge

\begin{tabular}{|c|c|c|c|c|}
\hline $\begin{array}{l}\text { centrée autour de } \\
\text { la traque d'un san- } \\
\text { glier monstrueux } \\
\text { nommé Trwyth } \\
\text { - un roi métamor- } \\
\text { phosé ainsi à cause } \\
\text { de ses péchés }{ }^{49} \text { - et } \\
\text { de sa harde, } \\
\text { vraisemblablement } \\
\text { issus de l'Autre } \\
\text { Monde }{ }^{50} \text {, qui rava- } \\
\text { gent le pays. } \\
\text { Ils sont chassés par } \\
\text { le roi Arthur, célè- } \\
\text { bre entre tous. } \\
\text { Ils sont finale- } \\
\text { ment tués ou ils } \\
\text { disparaissent sans } \\
\text { laisser de traces } \\
\text { dans la mer }{ }^{51} \text {. } \\
\text { À leur traque sont } \\
\text { aussi attachés deux } \\
\text { bœufs qui sont, } \\
\text { eux aussi, des rois } \\
\text { métamorphosés } \\
\text { à cause de leurs } \\
\text { péchés : Nynnio et } \\
\text { Peibio. } \\
\text { Ce dernier a } \\
\text { commis notamment } \\
\text { un inceste qu'il } \\
\text { cherche à cacher } \\
\text { en jetant à l'eau, } \\
\text { puis en brûlant la } \\
\text { mère et l'enfant né } \\
\text { de cet inceste. } \\
\text { À la fin de l'histoire, } \\
\text { Ysbaddaden, dont } \\
\text { la fille s'allie à ses } \\
\text { ennemis, est tué. }\end{array}$ & $\begin{array}{l}\text { Díl est engagé dans } \\
\text { une légende mytho- } \\
\text { logique } \\
\text { selon laquelle } \\
\text { Cairbre Músc } \\
\text { conquiert le trône } \\
\text { aux dépens de son } \\
\text { beau-père, avec } \\
\text { l'aide d'un roi } \\
\text { étranger. } \\
\text { Corc et son clan } \\
\text { sont traqués } \\
\text { comme des san- } \\
\text { gliers. }\end{array}$ & $\begin{array}{l}\text { Díl est engagé dans } \\
\text { une légende mytho- } \\
\text { logique } \\
\text { selon laquelle } \\
\text { Lughaidh mac Con } \\
\text { conquiert le trône } \\
\text { aux dépens de son } \\
\text { beau-père, avec } \\
\text { l'aide d'un roi } \\
\text { étranger. } \\
\text { Díl est un sanglier } \\
\text { et/ou est associé } \\
\text { avec la traque } \\
\text { d'une harde de san- } \\
\text { gliers monstrueux } \\
\\
\text { issus de l'Autre } \\
\text { Monde, } \\
\text { qui ravagent le } \\
\text { pays. } \\
\text { Ils sont chassés par } \\
\text { le roi Ailill et la } \\
\text { reine Meadhbh, } \\
\text { célèbres entre tous } \\
\text { et finalement ame- } \\
\text { nés à disparaître } \\
\text { sans laisser de } \\
\text { traces. }\end{array}$ & $\begin{array}{l}\text { est associé au } \\
\text { sanglier } \\
\text { nommé Triath, } \\
\text { le roi des sangliers, }\end{array}$ & $\begin{array}{l}\text { Triath signifie } \\
\text { roi, sanglier }\end{array}$ \\
\hline
\end{tabular}

49. Mal y cafas Culhwch Olwen in R. Bromwich, D. S. Evans, Culhwch and Olwen, op. cit., p. 38 : «C'était un roi mais Dieu l'a transformé en porc pour ses péchés " (trad. P.-Y. Lambert, Les quatre branches du Mabinogi et autres contes gallois du Moyen Âge, op. cit., p. 160).

50. Il vient " d'outremer ".

51. Précisément dans le Llyn Lliwan : Mal y cafas Culhwch Olwen in R. Bromwich, D. S. Evans, Culhwch and Olwen, op. cit., p. 40-41. 
Une troisième attribue le défrichage de la seule Magh Feimhin aux deux bœufs royaux, les nomme Fea et Mean, et les associe à une Díl Dealghan, fille de Golamh Míl - l'ancêtre des Milésiens - ou d'un obscur Lughmannar.

Le plus clair exposé de ces versions concurrentes se trouve dans la notule Magh Feimhin du Dinnsheanchas de Rennes :

«Trois fils de Mogach mac Dácháir, du clan de Brath mac Deatha : Feimhean, Feara et Fea. Ils amenèrent une cognée, une serpe et une bêche. Quand Feamhan bêchait, Feara bûcheronnait et Fea émondait; quand Fea bûcheronnait, Feara bêchait et Feimhean émondait : ils s'échangeaient ainsi [à tour de rôle] leurs outils et ils défrichèrent [ainsi] trois plaines : Magh Feimhin, Magh Feara et Magh Fea.

Ou encore, les deux boufs de Díl, fille de Lughmannar, expirèrent là. Leurs noms étaient Fea et Mean ${ }^{52}$ et c'est d'eux qu'a été formé celui de Magh Feimhin. Magh Fea, elle, tire son nom de Fea, fille d'Ealcmhar et épouse de Néid mac Ionnaoi.

En vérité, Feimhean et Feara, de la pure descendance de Death, ce sont eux qui ont défriché les bois pour en faire deux plaines ${ }^{53}$."

Pour embrouiller encore l'écheveau, l'histoire prêtée à Díl Dealghan l'associe non à deux bœufs mais à un seul. Elle est contée par les notices topologiques prétendant révéler l'origine du nom de Magh Breágh, la plaine côtière au nord du Leinster.

Celles-là proposent deux origines : la plaine tirerait son nom soit de Breágha mac Breoghain, soit d'un bœuf Breágha amené là par Díl :

"Magh Breágh : d'où vient ce nom? Ce n'est pas difficile à dire.

Breágha mac Breoghain fut l'aîné des enfants de Breoghan ${ }^{54}$ et c'est par lui que cette plaine fut essartée, et c'est pourquoi elle tire de lui son nom.

Ou alors c'est de Díl, fille de Lughmhannar, qui s'est enfuie du pays des Fir Falgha avec Tulchaointe, le druide de Conaire [Mór] ${ }^{55}$. À l'heure même de sa naissance, une vache avait mis bas un veau et dès lors la fillette s'était prise d'affection pour ce veau plus que pour tout autre car il était né en même temps qu'elle. Tulchaointe ne put donc l'enlever qu'en emmenant aussi le veau.

Tulchaointe était l'ami de la Mórríoghan ${ }^{56}$ : il la pria de transporter [magiquement] le troupeau à Magh Bolgaithe - c'est là le plus ancien nom de la plaine - et Breágha, le bœuf de Díl, aima cet endroit, de sorte que son

52. Cf. Sanas Chormaic 603 in K. Meyer, Sanas Cormaic, op. cit., p. 49 : « Feimhean : c'est Fea et Mean, autrement dit les deux rois des bovidés d'Irlande ".

53. Dinnsheanchas de Rennes 44 in W. Stokes, "The Prose Tales in the Rennes Dindsenchas ", art. cit., XV, p. 435-436. Cf. Dinnsheanchas bodléien 16 in W. Stokes, "The Bodleian Dindsenchas ", Folklore, III, p. 483-484; Dinnsheanchas du Leabhar Uí Maine 72 in E. J. Gwynn, "The Dindsenchas in the Book of Uí Maine ", Ériu, X, 1926-1928, p. 84; Dinnsheanchas métrique in E. J. Gwynn, The Metrical Dindsenchas, op. cit., III, p. 198 et 204 .

54. Breoghan est le père de Golamh Míl, souche des Milésiens déjà cités.

55. Le pays des Fir Falgha est l'île de Man; Conaire Mór est l'un des plus célèbres hautsrois de Tara : cf. D. Ó hÓgáin, Myth, Legend and Romance, op. cit., p. 99-100.

56. La Mórríoghan est une importante déesse irlandaise : cf. F. Le Roux-Guyonvarc'h, C. J. Guyonvarc'h, Morrigan-Bodb-Macha, Rennes, 1983. 
nom y est resté attaché et que c'est pour cela qu'on l'appelle [maintenant] Magh Breágh ${ }^{57}$."

\section{Déluge(s)}

Les confusions et rapprochements abusifs sautent aux yeux et seule l'association de Triath, de Díl et des deux bœufs royaux ${ }^{58}$ apparaît ici parallèle au matériel gallois.

- Le nom de Díl - comme sans doute celui de Dillus Barfog - est certainement apparenté au mot díle " océan ", lui-même utilisé au singulatif an Díleann pour désigner le Déluge qui a ennoyé la Terre entière ${ }^{59}$.

Or l'Irlande catholique s'est donné beaucoup de peine pour concilier ses croyances ancestrales préchrétiennes et la bible de sa nouvelle foi ${ }^{60}$.

Les premières imaginaient vraisemblablement la fin des temps - et l'intervalle de non-temps en celle-ci et le début d'un nouvel éon ${ }^{61}$ - comme la chute de l'axe cosmique soutenant le ciel de sorte que celui-là tombe sur la tête des résidents de ce bas monde, la confusion des éléments et le retour au chaos aqueux primordial.

En témoigne par exemple ${ }^{62}$ le serment du roi mythique Conchobhar mac Neasa lorsqu'il promet que seule la fin du monde pourrait l'empêcher de reprendre les femmes et le bétail d'Ulster razziés par ses ennemis :

Le ciel au-dessus de nous, la Terre sous nous et l'océan tout autour de nous. À moins que le ciel ne tombe, avec sa pluie d'étoiles, sur la Terre, que celle-ci soit brisée par un séisme et que la mer aux bleus sillons, riche en

57. Dinnsheanchas de Rennes 111 in W. Stokes, "The Prose Tales in the Rennes Dindsenchas ", art. cit., XVI, p. 62. Cf. Dinnsheanchas bodléien 2 in W. Stokes, "The Bodleian Dindsenchas ", art. cit., p. 470-471; Dinnsheanchas du Leabhar Uí Maine 7 in E. J. Gwynn, "The Dindsenchas in the Book of Uí Maine ", Ériu, X, 1926-1928, p. 84; Dinnsheanchas métrique in E. J. Gwynn, The Metrical Dindsenchas, op. cit., IV, p. 190-192.

58. La présence de Brighid répond sans doute à son autorité générale sur l'ensemble du règne animal, qu'elle a d'ailleurs transmise à Brigide, la sainte très catholique qui a christianisé son personnage : cf. C. Sterckx, Sangliers Père \& Fils, op. cit., p. 66-68.

59. P.S. Dinneen, Foclóir gaedhilge agus béarla, Dublin, 1934, p. 335.

60. Voir J. Carey, The Irish National Origin-Legend : Synthetic Pseudohistory, Dublin, 1994, A Single Ray of the Suni, Andover, 1999, p. 1-38.

61 . On peut y comparer les croyances des Germains sur la fin des temps, quand Surt enflammera tout l'univers et que la Terre s'enfoncera dans la mer, et sur la naissance d'un nouvel éon quand la Terre émerge de la mer et que les fils des dieux morts règnent sur un monde neuf (C. Sterckx, "Les eschatologies des Germains et des Celtes ", Ollodagos, XXI, 2007, p. 31-71) ou avec les croyances indiennes selon lesquelles la Terre est périodiquement réduite en cendres, les dieux et le ciel détruits, les trois mondes confondus en un seul océan... jusqu'à ce que Brahma se réveille de son sommeil, découvrant que le monde est vide, et que Visnu se dévoue pour faire émerger à nouveau la terre de l'Océan (C. Sterckx, Les dieux protéens des Celtes et des Indo-Européens, op. cit, p. 65-77).

62. Voir W. Sayers, " Ringing Changes on a Cosmic Motif ", Ériu, XXXVII, 1986, p. 99-117 pour d'autres analogies. 
frai, ne recouvre sa face, je ramènerai les vaches à leur étable et les femmes à leur foyer ${ }^{63}$.

Le confirment par ailleurs l'anecdote fameuse des Gaulois se vantant devant Alexandre III le Grand qu'ils ne craignent rien ni personne, si ce n'est que le ciel leur tombe sur la tête ${ }^{64}$; le conseil moqueur de Juvénal :

"Garde-toi $[\ldots]$ de l'axe du monde gaulois ${ }^{65}$;"

la parodie de Fortunat

" La grande colonne s'écroula et les cieux les écrasèrent ${ }^{66}$; " Olwen:

ou le proverbe gallois conservé par le vénérable Mal y cafas Culhwch

"Quand [ces] trois hommes sonnent du cor et que les autres se mettent à crier, on n'entendrait même plus le ciel tomber sur la Terre ${ }^{67}$."

En témoigne sans doute aussi le texte de Strabon :

"Les druides et d'autres avec eux affirment que les étincelles vitales et le monde sont impérissables et qu'un jour ne régneront plus que le feu et l'eau ${ }^{68}$. "

- L'équivalent le plus évident de ces concepts dans la bible chrétienne était évidemment le Déluge et c'est dès lors en toute bonne foi - au premier sens du terme! - que l'Irlande catholique a mêlé ses vieilles légendes au déluge biblique, non sans hésiter, tant son attachement à ses légendes était fort, à oser parfois contredire la Bible en affirmant que, contrairement à ce qu'enseigne celle-là, Noé et les siens n'ont pas été les seuls survivants au cataclysme mais qu'il y a en outre un être exceptionnel - éventuellement quadruplé pour répondre à son symbolisme de totalité ${ }^{69}$ - qui transcende

63. Táin bó Cúailnge in C. O'Rahilly, Táin bó Cúailnge from the Book of Leinster, Dublin, 1967, p. 112. Cf. H. d'Arbois de Jubainville, Etudes sur le droit celtique, Paris, 1895, I, p. 21 24; F. Le Roux-Guyonvarc'h, C.-J. Guyonvarc'h, Les druides, Rennes, 19864, p. 135-138.

64. Arrien, Anábasis Alexándrou I 4 6-8 in A. G. Roos, G. Wirth, Flauii Arriani quae exstant omnia, Leipzig, 1967, I, p. 9-10; Strabon, Geographiká VII 38 in H.L. Jones, The Georgaphy of Strabon, Londres, 1917-1932, III, p. 202. Cf. Aristote, Ethikà nikhomákheia III 77 in H. Rackham, Aristotle. The Nichomacheian Ethics, Londres, 1934, p. 158; voir aussi H. d'Arbois de Jubainville, Etudes sur le droit celtique, op. cit. I, p 20-21; C. Jullian, « La chute du ciel sur les Gaulois ", Revue des Études Anciennes, VIII, 1906, p. 259; F. Marco Simón, Milenarismo y percepción del tiempo entre los Celtas, in J. Mangas, S. Montero (éd.), El millenarismo, Madrid, 2001, p. 106-108.

65. Juvenal, Saturae VIII 116 in P. de Labriolle, F. Villeneuve, Juvénal. Satires, Paris, 1921, p. 106.

66. Fortunat, Carmina X 6 in F. Leo, Venanti Honori Clementiani Fortunati presbyteri italici opera poetica, Berlin, 1881, p. 238.

67. Mal y cafas Culhwch Olwen in R. Bromwich, D. S. Evans, Culhwch and Olwen, op. cit., p. 28; cf. P.-Y. Lambert, Les quatre branches du Mabinogi et autres contes gallois du Moyen Age, op. cit., p. 148. Pour d'autres allusions galloises à cette croyance : R. Bromwich, D. S. Evans, Culhwch and Olwen, op. cit., p. 137.

68. Strabon, Geographiká IV 4 4, in F. Lasserre, op. cit., p. 161.

69. Soit un "aux quatre coins du monde". 
les éons et conserve de l'un à l'autre la science divine et le principe de vie ${ }^{70}$. Voici par exemple ce qu'il dit de lui-même :

"Moi, j’ai été préservé

Par le fils de Dieu, protecteur des hommes,

Si bien que le Déluge me porta

Au sommet du lourd Tounthinna ${ }^{71}$.

Je suis resté un an sous le Déluge

Au fort de Tounthinna

$[\ldots]$

Je suis Fionntan Finn[eolach],

Fils de Bóchra. Je ne le cacherai pas :

Depuis le Déluge, ici-bas

Je suis un sage éminent et noble ${ }^{72}$.

Avant le noir Déluge, j’ai vécu

Quinze années.

Après le Déluge, Dieu m'a donné

Cinq mille cinq cents ans ${ }^{73}$."

Et Geoffrey Keating cite un vieux poème :

"Le nom des quatre, à bon droit,

Que Dieu garda saufs pendant le Déluge :

Fionntan, Firéan, Fors bon et noble

Et Annoith mac Eithiuir.

À Fors, en Orient, l'est fut donné;

À Firéan la froideur quand on n'a pas de vêtement [= le nord];

À Fionntan l'ouest bien délimité

Et à Annoith le sud.

Bien que les savants content cela,

Le canon véridique [= la Bible] ne le dit pas

Mais que seuls, dans l'arche, Noé, ses enfants

Et leurs épouses eurent la vie sauve ${ }^{74}$."

- Par ailleurs, le monde celte préchrétien ne concevait pas la résorption au chaos aqueux, marquant la fin de l'éon, sous l'aspect d'une pluie diluvienne ainsi que le formule la Bible :

"Tous les réservoirs du grand Abîme furent rompus et les ouvertures du ciel furent béantes. La pluie se déversa sur la Terre pendant quarante jours et quarante nuits ${ }^{75}$."

70. D'ailleurs assimilés l'un à l'autre, comme en toute bonne métaphysique...

71. Tounthinna (Tonn Tuine " La Vague des Vagues ") est un oronyme de Templecallow, en Munster : E. I. Hogan, Onomasticon goidelicum, Dublin, 1910, p. 658.

72. Suidhiughadh teallaigh Teamhrach 9 in R. I. Best, "The Settling of the Manor of Tara ", Ériu, IV, 1910, p. 121-172.

73. Ársaidh sín, a eoin Accla 8 in K. Meyer, « The Colloquy between Fintan and the Hawk of Achill ", in O. Bergin et al., Anecdota from Irish Manuscripts, op. cit., p. 24-39.

74. Geoffrey Keating, Foras feasa ar Éirinn I 5 in D. Comyn, P. S. Dinneen, The History of Ireland by Geoffrey Keating, op. cit., I, p. 148. Sur tout cela : C. Sterckx, Les dieux protéens des Celtes et des Indo-Européens, op. cit., p. 21-38.

75. Genèse VII 11-12 (traduction œcuménique). 
Il l'imaginait comme une submersion générale causée par un débordement, véritable éruption de la source cosmique ${ }^{76}$, et les scénarios qui s'en laissent reconstituer s'avèrent remarquablement concordants au-delà de leur diversité apparente.

- Le plus célèbre est celui qui se laisse reconstituer en Irlande.

Celui-là dit que, au pied de l'axis mundi, sourd la source cosmique d'où émanent et où refluent toutes les eaux vives - vivifiantes - du monde.

"[Patrice] vint à la source de Fionnmhagh ["la Plaine Brillante"], qu'on appelle Slán ["Parfaite, Totale"], car il lui avait été dit que les druides la vénéraient et lui offraient des sacrifices comme à une divinité. Elle était quadrangulaire et il s'y trouvait une bonde en pierre [également] quadrangulaire. Et l'eau sourdait au-dessus de la bonde ${ }^{77} \ldots$ ".

"Cormac voit dans le jardin [de l'Autre Monde] une source brillante de laquelle naissent cinq fleuves. Les hôtes boivent l'eau à la ronde. Les neufs coudriers de Búanann ${ }^{78}$ se dressent au-dessus d'elle. Ces coudriers pourpres laissent choir leurs noisettes dans la source et les cinq saumons qui s'y trouvent les ouvrent et laissent flotter leurs coques au fil des fleuves ${ }^{79}$."

"C'est une source auprès de laquelle se dressent les neuf coudriers de l'inspiration poétique. Leurs fruits, leurs fleurs et leurs feuilles tombent en même temps dans la source et la colorent royalement de pourpre. Alors le

76. C'est d'ailleurs là un héritage majeur reçu d'un très lointain passé car cette croyance se retrouve d'un bout à l'autre de l'espace culturel indo-européen : G. Dumézil, "Le puits de Nechtan ", Celtica, VI, 1963, p. 50-61, Mythe et épopée, Paris, 1968-1973, III, p. 21-89; C. S. Littleton, "Poseidon as a Reflex of the Indo-European « Source of Waters " God ", Journal of Indo-European Studies, I, 1973, p. 423-440.; P. K. Ford, "The Well of Nechtan and la Gloire Lumineuse ", in G. J. Larson (ed.), Myth in Indo-European Antiquity, Berkeley, 1974, p. 67-74; D. Briquel, J.-L. Desnier, « Le passage de l'Hellespont par Xerxès ", Bulletin de l'Association Guillaume Budé, 1983, p. 22-30; J. Puhvel, « Aquam extinguere ", Journal of Indo-European Studies, I, 1973, p. 379-386, Comparative Mythology, Baltimore, 1987, p. 277-283; M. Meulder, "Le feu dans l'eau en Sicile ", Ollodagos, XI, 1998, p. 89-109; D. Briquel, " Vieux de la Mer grecs et Descendants des Eaux indo-européens ", in R. Bloch (éd.), Recherches sur les religions de l'Antiquité classique. Genève, 1980, p. 141-158, "Sur un passage d'Hérodote : prise de Babylone et prise de Veies ", Bulletin de l'Association Guillaume Budé, 1981, p. 293-306, « Le thème indo-européen du feu dans l'eau ", in G. Capdeville (éd.), L'eau et le feu, Paris, 2004, p. 11-23; J.-L. Desnier, "Le feu dans l'eau ", in G. Capdeville (éd.), L'eau et le feu, op. cit., p. 291-302; C. Sterckx, Dieux d'eau : Apollons celtes et gaulois, Bruxelles, 1996, « Le feu dans les eaux de l'Apollon gaulois ", in G. Capdeville (éd.), L'eau et le feu, op. cit., p. 303-319; L'œil, la main, le pied : ordalies trifonctionnelles, 2007 (à paraître).

77. Tíreachán, Collectanea in L. Bieler, The Patrician Texts in the Book of Armagh, Dublin, 1979, p. 150, cité ici d'après la traduction anglaise de J. Carey, " St Patrick, the Druids and the End of the World ", History of Religions, XXXVI, 1996-1997, p. 44.

78. Búanann est l'un des noms de la déesse-mère Dana: C. Marstrander et al., (Contributions to a) Dictionary of the Irish Language, Dublin, 1913-1975, p. B227-228. Cf. Sanas Chormaic 104 in K. Meyer, Sanas Cormaic, op. cit., p. 11.

79. Eachtra Chormaic i dTír Tairngire 35 in W. Stokes, "The Irish Ordeals, Cormac's Adventure in the Land of Promise and the Decision as to Cormac's Sword ", in W. Stokes, E. Windisch, Irische Texte, op. cit., III, 1891, p. 195. Cf. Dinnsheanchas métrique in E.J. Gwynn, The Metrical Dindsenchas, op. cit., II, p. 292-294. 
saumon mâche les noisettes et leur jus donne sa pourpre à son ventre. Et sept fleuves de science en émanent et y refluent ${ }^{80}$."

"C'est le bonheur de la science poétique totale qu'accordent les neuf coudriers de la Seaghais, au séjour des dieux : leurs noisettes tombent, grosses comme des têtes de béliers, et elles sont emportées par la Boyne, à la vitesse de chevaux au galop, tous les sept ans à la mi-juin (= au solstice d'été $)^{81}$. "

"Quinze noms sont donnés au fleuve qui sourd du séjour de Neachtan et atteint le Paradis d'Adam [...] : Seaghais est son nom au séjour de Neachtan [...], Severn en bonne terre saxonne, Tibre dans l'empire romain, Jourdain au loin en Orient et le large Euphrate [...]; il est le Tigre au Paradis [...] et du Paradis il revient ici au séjour de Neachtan ${ }^{82}$."

"L'inspiration poétique totale " n'est évidemment pas à prendre ici comme le simple talent du rimailleur : c'est l'omniscience divine absolue comme le montrera d'ailleurs la suite du dossier. La source cosmique vivifie donc le monde parce que ses eaux contiennent cette science divine dont est aussi détenteur le Saumon de Science ${ }^{83}$ qui y nage et qui y absorbe régulièrement les noisettes de science ${ }^{84}$.

Source, noisettes et saumon ont un gardien, Ferfhí, et il est dit que certains héros sont parvenus à le capturer.

Une légende raconte comment Cúchulainn harponne un jour le Saumon de Science et capture le nain Seanbheag Ó hEbrice "Petit Vieux fils du Saumon Tacheté " qui poursuivait, dans une minuscule nacelle en bronze, le saumon et les noisettes de science. Il lui extorque un vêtement magique qui protège du feu et de l'eau ainsi que de la vieillesse ${ }^{85}$, puis un bouclier et un javelot qui rendent invincible... mais, peu après, le nain réussit à saisir sa rote et à en pincer les cordes : leur son endort le héros et Seanbheag réussit à s'enfuir avec le saumon et les merveilles qu'il avait dû céder ${ }^{86}$.

80. Dinnsheanchas de Rennes 59 in W. Stokes, "The Prose Tales in the Rennes Dindsenchas ", art. cit., XV, p. 456. Un ou neuf (triple triplication intensive) coudriers, un ou cinq saumons (correspondant aux sages aux quatre coins du monde plus un pour le centre), quatre (plus la source centrale en cinquième), cinq ou sept fleuves qui naissent de la source : ce sont évidemment là des chiffres symboliquement interchangeables, portant tous valeur de totalité

81. Mo choire cóir II in P. L. Henry, " The Cauldron of Poesy ", Studia Celtica, XIV-XV, 19791980, p. 114-128. Cf. L. Breatnach, "The Caldron of Poesy ", Ériu, XXXII, 1981, p. 45-93; P. K. Ford, Ystoria Taliesin, op. cit., p. 27-28.

82. Dinnsheanchas métrique in E. J. Gwynn, The Metrical Dindsenchas, op. cit., III, p. 26-28.

83. Eventuellement quintuplé en cinq clones.

84. Cf. A. J. Hughes, "Some Aspects of the Salmon in Gaelic Tradition Past and Present ", Zeitschrift für Celtische Philologie, XLVIII, 1996, p. 17-28.

85. Ne faut-il pas ici l'entendre comme une protection face à l'eschatologie, assurant à son détenteur la faculté de transcender celle-là?

86. Cómhrac Chonchulainn re Seanbheag Ó hEbhrice ó Seaghais in K. Meyer, "Anecdota from the Stowe MS N 992 ", Revue Celtique, VI, 1883-1885, p. 184. Cf. E. J. Gwynn, " An Old-Irish Tract on the Privileges and Responsabilities of Poets ", Ériu, XIII, 1942, p. 26-27. La version qui remplace Seanbheag par Neachtan Ealcmhar et qui laisse Cúchulainn 
Une autre a déjà été évoquée, qui met à l'origine des démêlés de Lughaidh mac Con et de Díl la capture du gardien de la source et qui attribue à ce dernier le nom - particulièrement significatif, comme il apparaîtra plus loin - de Ferfhí.

« Deimhne partit pour apprendre la poésie auprès de Finnéigeas, qui vivait sur les rives de la Boyne [...]. Finnéigeas y vivait depuis sept ans en guettant le saumon de Linn Féich parce qu'il lui avait été prédit que rien ne resterait inconnu de celui qui le mangerait. Le saumon fut pris et Deimhne fut chargé de le cuire, avec défense par le poète d'en manger une seule miette. Après l'avoir fait cuire, le garçonnet apporta le saumon. "N'en as-tu rien mangé, gamin?" interrogea le poète. "Non", répondit-il, "mais j'y ai brûlé mon pouce que j'ai ensuite mis dans ma bouche". "Quel est ton nom, petit?" questionna le poète. "Deimhne" dit-il. "Ton nom sera désormais Fionn, mon garçon. C'est à toi qu'il a été imparti de manger le saumon et, en vérité, tu es le Fionn." Le garçonnet mangea alors la saumon et c'est cela qui a donné la connaissance [absolue] à Fionn : chaque fois qu'il mettait son pouce en bouche ${ }^{87}$ et chantait le téinm laodha ${ }^{88}$, tout ce qu'il voulait savoir lui était révélé ${ }^{89}$."

C'est là une histoire célèbre dans le monde gaélique, encore vivante aujourd'hui dans la tradition orale, et certaines versions remplacent la bénévolence et la rapide résignation de Finnéigeas par une rancœur meurtrière que Deimhne Fionn réussit à découvrir et à prévenir en suçant son pouce $^{90}$.

Reste encore la plus célèbre légende irlandaise attachée à la source cosmique $^{91}$.

maître du saumon n'est pas convaincante : C. Sterckx, Les dieux protéens des Celtes et des Indo-Européens, op. cit., p. 10.

87. Il existe plusieurs représentations anciennes de Fionn suçant son pouce de science : A. Kingsley Porter, "A Sculpture at Tanderagee ", Burlington Magazine, LXV, 1934, p. 227228; H. R. E. Davidson, "The Seer's Thumb ", in H. R. E. Davidson, The Seer in Celtic and Other Traditions, Edimbourg, 1990, p. 75-76.

88. Sur cette incantation divinatoire : F. Le Roux-Guyonvarc'h, " La divination chez les Celtes ", in A. Caquot, M. Leibovici (éd.), La divination, Paris, 1968, p. 244-245; F. Le RouxGuyonvarc'h, C. J. Guyonvarc'h, Les Druides, op. cit., p. 181-183.

89. Macgnímratha Fhinn 17-18 in K. Meyer, "Magnimratha Finn ", in Revue Celtique, V, 1881-1883, p. 201. Cf. R. D. Scott, The Thumb of Knowledge, New York, 1930; J. F. Nagy, "Liminality and Knowledge in Irish tradition ", Studia Celtica, XVI-XVII, 1981-1982, p. 139140, The Wisdom of the Outlaw, Berkeley, 1985; H. R. E. Davidson, "The Seer's Thumb ", in H. R. E. Davidson ed., The Seer in Celtic and Other Traditions, op. cit., p. 70-71; P. K. Ford, Ystoria Taliesin, Cardiff, 1992, p. 17-20; M. Freeman, "Eating the Salmon of Wisdom ", Parabola, XXII, 1997, p. 10-15.

90. J. F. Campbell, Popular Tales of the West Highlands, Londres, 1890-1893, III, p. 348-350; E. Ó Muirgheasa, " An dóigh a chuaidh Fionn i dtréis ", Béaloideas, I, 1927-1928, p. 405-410; D. Hyde, "Báirne Mór ", Béaloideas, III, 1931-1932, p. 194-195, 1936; J. Delargy, Leabhar Shéain I Chonaill, Dublin, 1948; etc. Cf. R. D. Scott, The Thumb of Knowledge, op. cit., p. 7581; D. Ó hÓgáin, Myth, Legend and Romance, op. cit., p.216-217.

91. Aux textes dont des extraits sont cités ci-dessous, ajouter Dinnsheanchas bodléien 36 in W. Stokes, "The Bodleian Dindsenchas ", art. cit., p. 500; Dinnsheanchas du Leabhar Uí Maine 8 in E. J. Gwynn, "The Dindsenchas in the Book of Uí Maine ", art. cit., p. 76. 
«Il y eut un fameux roi d'Irlande, de la race des Tuatha Dé Danann (= des dieux), dont le nom était Eochaidh Ollathair. On l'appelait aussi le Daghdha "le Bon Dieu" [...]. [Neachtan] Ealcmhar du Brugh ${ }^{92}$ avait une épouse du nom d'Eithne, qu'on appelait aussi Bóinn "Vache Blanche ${ }^{93}$ ". Le Daghdha s'éprit de désir pour elle. Il envoya alors Ealcmhar faire un long voyage chez Breas mac nEaladhan à Magh Inis. Quand Ealcmhar se mit en route, le Daghdha plaça sur lui de puissants charmes pour l'empêcher de rentrer à temps : il recula la tombée de la nuit et empêcha Ealcmhar de ressentir la faim ni la soif. Il le fit longtemps traîner ainsi : neuf mois qui lui parurent ne durer qu'un seul jour, car Ealcmhar avait annoncé qu'il rentrerait avant le crépuscule. Pendant ce temps-là, le Daghdha copula avec l'épouse d'Ealcmhar et elle lui donna un fils du nom d'Aonghus. Quand Ealcmhar rentra, son épouse était remise des peines de l'accouchement et il ne se rendit pas compte qu'elle avait fauté en copulant avec le Daghdha. Cependant, le Daghdha emmena son fils pour qu'il fût élevé dans la maison de Midhir, à Bri Léith de Teathbha. Aonghus y fut élevé pendant neuf ans ${ }^{94}$."

\section{Mais Eithne Bóinn veut totalement effacer sa faute :}

Neachtan [Ealcmhar] mac Labhraid Lorc avait, je l'affirme [Eithne] Bóinn pour épouse. Il possédait dans son domaine une source secrète d'où émanaient toutes sortes de maux mystérieux. Nul ne pouvait en regarder le fond sans que ses yeux n'en éclatassent : qu'on la contournât par la gauche ou la droite, on ne pouvait échapper à cette mutilation. Nul n'osait en approcher sauf Neachtan et ses trois échansons dont voici les noms célèbres : Fleasc, Lámh et Luath. C'est là que vint un jour Bóinn, infatuée par son noble orgueil : à la source intarissable pour en éprouver la vertu ${ }^{95}$.

"Bóinn partit en hâte de chez elle : elle était sûre que sa faute serait cachée si elle réussissait à s'y baigner ${ }^{96}$."

"C'est là que jaillit la source de la Seaghais [...]. Quiconque y vient avec un mensonge n'en revient pas sans mutilation [...]. "J'irai à la belle source de la Seaghais pour que ma chasteté ne puisse pas être mise en question. Je ferai trois fois le tour de la source de vie, véridique, dans le sens contraire au Soleil" [pensa Eithne Bóinn] ${ }^{97}$."

92. La résidence de Neachtan Ealcmhar est le Brugh na Bóinne, c'est-à-dire le site des trois grands tumulus néolithiques de Dowth, Knowth et Newgrange, en Meath.

93. Sur ce théonyme : P. de Bernardo Stempel, «Continental Celtic ollo : Early Welsh (h)ol(l), Olwen and Culhwch", Cambrian Medieval Celtic Studies, XLVI, 2003, p. 127. L'élément uind- qui se retrouve dans les noms de Finnéigheas, Deimhne Fionn, Fionntan, Bóinn... porte un sens premier « blanc, brillant, beau " mais aussi un sens « illumination, vision, connaissance " encore attesté en irlandais (fionnacht, fionnachtain). La rivière Boyne est donc, plus que la "Vache Blanche ", "la Vache (= la rivière) qui apporte la Connaissance, l'Illumination " : D. Ó hÓgáin, " The River Boyne and the Ancient Seers ", art. cit., p. 21-28.

94. Tochmarch Éadaoin I 1-2 in O. Bergin - R.I. Best, « Tocmarc Etaine ", Ériu, XIII, 1938, p. 145.

95. Dinnsheanchas métrique in E. J. Gwynn, The Metrical Dindsenchas, op. cit., III, p. 1903, p. 26-28.

96. Dinnsheanchas métrique in E. J. Gwynn, The Metrical Dindsenchas, op. cit., III, p. 1903, p. 38.

97. Cinaodh ó hArtacáin, Seacht o.f.n. 72-74 in L. Gwyn, "Cináed úa HArtacáin’s Poem on Brugh na Bóinne ", Ériu, VIII, 1914, p. 229. 
«Inconsidérément, [Eithne Bóinn] fit ainsi trois fois le tour de la source: trois vagues s'en élevèrent et causèrent sa mort ${ }^{98}$."

"Trois vagues se brisèrent sur [Eithne Bóinn] et lui arrachèrent une cuisse, une main et un œil. Fuyant sa honte [d'être dénoncée coupable], elle se tourna vers la mer et l'eau la poursuivit jusqu'à [former] l'embouchure de la Boyne ${ }^{99}$."

- Au Pays de Galles, la source cosmique est identifiée à la Severn, qui a sa source au mont Plynlimon tenu traditionnellement comme le centre du monde du microcosme gallois ${ }^{100}$ et qui est curieusement citée par les Irlandais comme l'une des émanations de leur Seaghais. Un saumon merveilleux y hante, jusque dans un légendaire encore vivant au siècle dernier ${ }^{101}$, les eaux du Llyn Lliwan, autrement dit l'estuaire de ce fleuve. Une très vieille tradition, clairement inspirée par le mascaret naturel de cet estuaire, compare remarquablement ce mascaret à deux rois se battant tête contre tête, comme des béliers, et prétend que si l'on s'approche de manière indue, l'eau s'y enfle, agresse les mal venus en gerbes furieuses et menace même de les engloutir ${ }^{102}$.

Mieux encore, la Severn aurait reçu son nom ${ }^{103}$ à la suite de la noyade dans ses eaux d'une princesse ayant commis un adultère avec un roi, exactement comme Eithne Bóinn noyée dans la rivière issue de la Seaghais et lui ayant laissé son nom ${ }^{104}$.

Et cette réputation de la Severn s'avère vraisemblablement très ancienne puisqu'un temple prestigieux, centré sur une source sacrée et dédié à un dieu Nodont, équivalent mythologique et linguistique exact du Neachtan irlandais, y a été édifié depuis au moins le milieu du quatrième siècle ${ }^{105}$.

98. Dinnsheanchas métrique in E. J. Gwynn, The Metrical Dindsenchas, op. cit., III, p. 32. 99. Dinnsheanchas de Rennes 19 in W. Stokes, "The Prose Tales in the Rennes Dindsenchas ", art. cit., XV, p. 315. De telles ordalies sont connues en Gaule et dans tout le monde indo-européen : cf. C., Sterckx, L'œil, la main, le pied : ordalies trifonctionnelles, 2007 (à paraître).

100. A. D. Rees, B. Rees, Celtic Heritage, op. cit., p. 173-176; C. Sterckx, " Le temps et le non-temps des Celtes : pourquoi la nuit avant le jour? ", in V. Pirenne-Delforge, Ö. Tunca ed., Représentations du temps dans les religions, Genève, 2003, p. 252-253.

101. I. D. Ifans, "Chwedl yr anifeiliaid hynaf ", Bulletin of the Board of Celtic Studies, XXIV, 1970-1972, p. 461-464

102. Nennius, Historia Britonum, 68-69 in J. Morris, Nennius British History and the Welsh Annals, Chichester, 1980, p. 81. Cf. Geoffrey de Monmouth, Historia regum Britanniae 150 in N. Wright, The Historia regum Britanniae of Geoffrey of Monmouth, op. cit., p. 106. Voir aussi G. L. Hamilton, "Storm-Making Springs, Rings of Invisibility and Protection ", Romanic Review, II, 1911, p. 355-375; G. Milin, " Geoffroy de Monmouth et les merveilles du lac de Llinligwan ", Cahiers de Civilisation Médiévale, XXXVIII, 1995, p. 173-183; C. Sterckx, Dieux d'eau: Apollons celtes et gaulois, op. cit., p. 10.

103. Sa forme ancienne, Sabrina, se retrouve aussi comme hydronyme d'un affluent de l'Escaut : l'actuelle Zeveren qui rejoint le fleuve à Gand (Flandre Orientale).

104. C. Sterckx, Dieux d'eau : Apollons celtes et gaulois, op. cit., p. 10-11. Cf. G. W. Goetinck, "Sabrina et Sulis ", Études Celtiques, XXXV, 2005, p. 293-312.

105. R. E. M. Wheeler, T. V. Wheeler, The Excavation of the Prehistoric, Roman and Post Roman Temple in Lydney Park, Gloucestershire, Oxford, 1932 ; P. J. Casey, B. Hoffmann, 
Il n'est sans doute pas anodin qu'on y ait mis au jour un relief en bronze représentant un pêcheur harponnant un saumon ${ }^{106}$ !

La pêche du Saumon de Science débouchant sur l'acquisition de l'omniscience par Deimhne Fionn connaît de fait un parallèle remarquable en Galles, d'autant plus remarquable que le premier nom du protagoniste, Gwion, est linguistiquement identique à celui de Ferfhí irlandais rencontré significativement ci-dessus : tous deux sont formés sur *uiso- " poison ${ }^{107}$ " : il s'agit de la fameuse légende de Taliesin, dont la meilleure version semble être offerte par Llywelyn Siôn, à la fin du seizième siècle ${ }^{108}$.

La magicienne Cyrridwenn, épouse de Tegid Moel, a accouché de la plus belle fillette de Grande-Bretagne, Crairfyw, et du garçonnet le plus laid du monde, Morfrân " Grand Corbeau ». Celui-là est si repoussant qu'elle estime nécessaire de racheter sa laideur en lui assurant l'inspiration poétique et la connaissance suprême.

Grâce à sa magie, elle prépare alors un bouillon merveilleux : à condition de ne pas interrompre son ébullition pendant un an et un jour, il fournira la quintessence du savoir absolu. Et pour assurer le respect de la condition, elle charge un aveugle, Dallmor Dallmaen, d'entretenir le feu sous le chaudron et un page, Gwion Bach, de remuer régulièrement le bouillon ${ }^{109}$.

Mais elle leur abandonne tout le souci et, en son absence, voilà finalement que trois gouttes brûlantes jaillissent du bouillon et viennent éclabousser le pouce de Gwion ${ }^{110}$ : en un réflexe naturel, il le porte aussitôt à sa bouche pour en sucer la douleur... et il obtient pour lui la science absolue car ce sont précisément ces trois gouttes-là qui l'ont concentrée!

"Excavations at the Roman Temple in Lydney Park, Gloucestershire, in 1980 and 1981 ", Antiquaries Journal, LXXIX, 1999, p. 81-143.

106. R. E. M. Wheeler, T. V. Wheeler, The Excavation of the Prehistoric, Roman and Post Roman Temple in Lydney Park, Gloucestershire, op. cit., p. 100-104.

107. E. P. Hamp, "Gwion and Fer Fí ", Ériu, XXIX, 1978 p. 152-153. Cette étymologie commune tend à confirmer la vénérabilité du détail gallois selon lequel une seule goutte du chaudron de Cyrridwen contient l'omniscience et le reste un poison mortel. Par ailleurs, nous avons essayé de démontrer l'équivalence sémantique et mythologique du chaudron merveilleux, contenant la science divine qui est aussi la vie, et de la tête coupée (C. Sterckx, La mutilation rituelle des ennemis et les concepts de l'âme chez les Celtes préchrétiens, Paris 2005, p. 113-135) : n'est-ce qu'un hasard si, en Grèce, le tête coupée de Méduse contient à la fois un principe de vie et un poison mortel (Apollodore, Bibliothéke: III 103 in J. G. Frazer, Apollodorus. The Library, Londres, 1946, II, p. 16; Euripide, Ion 9991015 in L. Parmentier, H. Grégoire, Euripide. Tragédies III, Paris, 1923, p. 224. Cf. M. HalmTisserant, Cannibalisme et immortalité, Paris, 1993, p. 78, n. 8).

108. Hanes Taliesin in P. K. Ford, "A Fragment of the Hanes Taliesin by Llewelyn Siôn ", Études Celtiques, XIV, 1975, p. 451-460.

109. Sur cette réunion de l'aveugle Dallmor, du laid Morfran et de Gwion Taliesin : P. K. Ford, "The Blind, the Dumb and the Ugly: Aspects of Poets and their Crafts in Early Ireland and Wales ", Cambridge Medieval Celtic Studies, XIX, 1990, p. 40.

110 . Une version secondaire donne des détails légèrement différents : Gwion aurait sciemment accaparé les trois gouttes de science : cf. J. Wood, « The Folklore Background of the Gwion Bach Section of Hanes Taliesin ", Bulletin of the Board of Celtic Studies XXIX, 1981-1982, 621-634; mais le dossier comparatif lui enlève toute valeur. 
Sa première révélation est heureusement celle du sort que Cyrridwen lui réservera si elle le prend, et il s'enfuit à toutes jambes. Quant au chaudron, il explose car, hormis les trois gouttes merveilleuses, le reste du bouillon constitue un poison si violent qu'il n'y peut résister.

Arrivant alors pour recueillir le fruit de sa préparation, Cyrridwen enrage d'en être flouée et sa lance à la poursuite du page. Quand il la voit derrière lui, celui-ci se transforme en lièvre : Cyrridwen se change en lice et lui donne la chasse; arrivant près d'une rivière, Gwion y plonge et se transforme en poisson: Cyrridwen le presse sous la forme d'une loutre; Gwion se transforme en oiseau et s'envole : Cyrridwen se change en faucon et le traque dans les airs; serré de près et épuisé, Gwion aperçoit un tas de froment battu sur une aire et il s'y dissimule sous la forme d'un grain de blé : Cyrridwen se métamorphose en poule noire, le picore et l'avale.

Revenue à la forme humaine, Cyrridwen se trouve alors enceinte du petit Gwion. Après neuf mois de gestation, elle le (re)met au monde sous les traits d'un enfantelet si joli qu'elle n'a pas le cour de le tuer : elle l'enferme dans un sac en cuir qu'elle jette à la mer...

Le nouveau-né est sauvé deux jours plus tard ${ }^{111}$. En ce temps-là en effet, un prince gallois, Gwyddno Garanhir, aviat un fils, Elffin, qui était le garçon le plus malchanceux qui fût. Gwyddno possédait aussi une senne merveilleuse qui lui rapportait cent livres de poissons d'un seul coup à chaque calende de mai. Dans l'espoir de porter enfin chance à son fils, il lui confie la senne cette année-là... et Elffin n'en retire pour sa part que le sac en cuir contenant le bébé de Cyrridwen. Quand il l'ouvre, la fraîcheur du bambin le fait s'écrier " le beau front [= visage] que voilà! ", ce qui assure à l'enfançon le nom de Taliesin "Beau Front ${ }^{112}$ ".

Ici s'arrête le texte de Llywelyn Siôn. La suite de l'histoire se trouve dans une version plus ancienne mais moins précise : l'Ystoria Taliesin d'Ellis Gruffydd ${ }^{113}$.

Elle conte comment désormais la chance sourit sans discontinuer à Elffin et prête à Taliesin un long poème dans lequel il révèle qu'il est né au commencement des temps, a transcendé tous les âges et subsistera jusqu'à la fin des temps :

" J'étais[déjà] avec Dieu dans le ciel

Quand Lucifer est tombé aux abysses de l'Enfer;

J'ai été porte-bannière devant Alexandre [le Grand];

Je connais les noms des étoiles, depuis le nord jusqu'au sud;

J'ai été le Tétragramme à Caer Gwydion;

111. Le texte de Llywelyn Siôn énonce un séjour de onze jours en mer mais il est contredit par l'ensemble de la tradition (P. K. Ford, « A Fragment of the Hanes Taliesin by Llewelyn Siôn ", art. cit., p. 453) : il y a là probablement confusion entre ii et 11.

112. Sur ce nom : P. K. Ford, "The Blind, the Dumb and the Ugly: Aspects of Poets and their Crafts in Early Ireland and Wales ", art. cit., p. 35.

113. Ystoria Taliesin in P.K. Ford, Ystoria Taliesin, op. cit., p. 69-86. 
J'étais en Canaan quand Absalon a été tué;

J'ai ensemencé la vallée de Hébron;

J'ai été à la Cour de Dôn avant la naissance de Gwydion;

J'étais [déjà] un patriarche pour Enoch et pour Elie;

J'ai été maître d'œuvre de la tour de Nemrod;

J'étais sur la croix du fils de Dieu, le miséricordieux,

J'ai été trois fois dans la prison d'Aranrhod;

J'étais dans l'arche avec Noé et Alpha (= Dieu le Père);

J'ai vu la destruction de Sodome et de Gomorrhe;

J'ai été en Afrique avant la fondation de Rome :

J'y suis venu avec les survivants de Troie (= Enée et ses compagnons à la Cour de Didon);

J'étais avec mon seigneur [Jésus] dans la crèche du bœuf et de l'âne;

J'ai soutenu Moïse à travers l'eau du Jourdain; J'ai été avec Marie-Madeleine au ciel;

J'ai obtenu l'inspiration poétique du chaudron de Cyrridwen;

J'ai été le harpiste de Lleon Llychlyn ${ }^{114}$;

J'ai été à Gwynfryn, à la Cour de Cynfelin ${ }^{115}$

Dans les fers pendant un an et un jour.

C'est moi qui ai révélé au pays (=la Grande-Bretagne) la Trinité (= la religion catholique)

Et qui l'ai transmise au monde entier.

Et je resterai sur cette Terre jusqu'à la fin du monde.

Personne ne sait ce dont je suis fait : chair ou poisson?

J'ai été pendant neuf mois dans le sein de la magicienne Cyrridwen;

J'ai été jadis Gwion Bach, je suis maintenant Taliesin

$[\ldots]$

Le prophète Jean m'a appelé Merlin,

Maintenant tous les rois me nomment Taliesin ${ }^{116}$."

Si ces textes sont relativement tardifs, l'ancienneté de la légende est assurée par de nombreuses allusions dans des œuvres beaucoup plus anciennes.

La laideur de l'infortuné Morfrân est évoquée dans les Pedwar marchog ar hugain llys Arthur, qui la disent telle que nul n'osait lui refuser quoi que ce fût, et par le Mal y cafas Culhwch Olwen, qui la dit telle qu'aucun ennemi n'osa tourner ses armes contre lui à la bataille de Camlann, en précisant qu'il était velu comme un cerf ${ }^{117}$.

114. Ou " des légions de Scandinavie " (ibid., p. 117)?

115. Cynfelin est le nom gallois de Cunobélinos, le dernier grand roi de la GrandeBretagne préromaine, et Gwynfryn est identifié à sa résidence, censée s'être trouvée à l'emplacement de l'actuelle cathédrale Saint-Paul, à Londres.

116. Ystoria Taliesin in P. K. Ford, Ystoria Taliesin, op. cit., p. 76-78. Les deux derniers vers de notre citation, ainsi disposés pour faciliter la compréhension, sont en fait les premiers du texte original.

117. Pedwar marchog ar hugain llys Arthur in R. Bromwich, Trioedd Ynys Prydain, op. cit., p. 267; Mal y cafas Culhwcu Olwen in R. Bromwich, D. S. Evans, Culhwch ac Olwen, op. cit., p. 8-9. 
La beauté tout aussi exceptionnelle de Crairfyw lui a valu une place dans les Trioedd Ynys Prydain en tant que l'une des trois " filles à la beauté éclatante " de lî̀le de Grande-Bretagne ${ }^{118}$.

Le chaudron d'inspiration de Cyrridwen est cité dès le tournant du treizième siècle par Llywarch Prydydd y Moch ${ }^{119}$ et, un peu plus tard, par Casnodyn ${ }^{120}$. Il est attribué à Gwion Bach par Dafydd ab Edmwnt ${ }^{121}$ et par William Llŷr ${ }^{122}$.

L'affirmation prêtée par le Hanes Taliesin à son héros qu'il est une réincarnation de Merlin est commentée par Ellis Gruffydd, qui atteste qu'elle était bien reçue dans le légendaire gallois ${ }^{123}$ :

" Diverses rumeurs courent dans l'opinion, qui est elle-même divisée. Certains croient et maintiennent fermement que Merlin était un esprit sous la forme d'un homme et qu'il est resté dans cet état depuis le temps de Vortigern jusqu'aux débuts du roi Arthur, époque à laquelle il disparut. Plus tard, cet esprit renaquit au temps de Maelgwn de Vénédotie et il fut appelé alors Taliesin, celui dont on dit qu'il vit encore dans une cité du nom de Caer Sidia. Puis il naquit une troisième fois au temps de Morfryn Brych ab Esylt, dont on dit qu'il était le fils, et à cette époque il fut appelé Merlin l'Egaré. Et depuis lors jusqu'à nos jours, il est dit qu'il vit à Caer Sidia, d'où certains croient fermement qu'il surgira à nouveau une fois avant le jour du Jugement $\left[\right.$ Dernier] ${ }^{124}$."

Dernier détail significatif - du moins pour le présent dossier ${ }^{125}-$, un autre nom de la mère de Taliesin apparaît plus révélateur que celui de Cyrridwen : le De sancto Iudicaelo rege d'Ingomar le dit en effet fils de Dôn, autrement dit la Terre-Mère dont sont nés tous les dieux ${ }^{126}$.

Si Gwion-Taliesin-Merlin apparaît bien comme primordial et " antédiluvien ", le thème de l'éruption de la source de vie et d'omniscience se trouve

118. Trioedd Ynys Prydain 78 in R. Bromwich, Trioedd Ynys Prydain, op. cit., p. 208. Elle est aussi régulièrement évoquée dans la poésie ancienne : ibidem, p. 315-316.

119. J. Morris-Jones, T. H. Parry-Williams, Llawysgrif Hendregadredd, Cardiff, 1933, p. 305.

120. J. G. Evans, Poetry from the Red Book of Hergest, Lampeter, 1911, p. 1241.

121. T. Roberts, Gwaith Dafydd ab Edmwnt, Bangor, 1914, p. 143.

122. T. Parry, The Oxford Book of Welsh Verses, Oxford, 1962, p. 205.

123. Le rapport d'un espion au service des Tudor note pareillement que les Gallois passaient leurs dimanches à écouter des légendes sur Taliesin et Merlin (I. Williams, " Hen chwedlau ", Transactions of the Honourable Society of Cymmrodorion, 1946-1947, p. 28).

124. T. Jones, " The Story of Myrddin and the Five Dreams of Gwendydd in the Chronicle of Elis Gruffyd ", Etudes Celtiques, VIII, 1958-1959, p. 320-321; P. K. Ford, "The Death of Merlin in the Chronicle of Elis Gruffydd ", Viator, VII, 1976, p. 379-390. Sur Caer Sidia, ou mieux Caer Sidi, comme désignation de l'Autre Monde : C. Sterckx, Les dieux protéens des Celtes et des Indo-Européens, op. cit., p. 48.

125. Pour une étude plus complète de la figure de Taliesin et de ses parallèles : C. Sterckx, Les dieux protéens des Celtes et des Indo-Européens, op. cit.

126. Tout comme les dieux irlandais sont appelés collectivement Tuatha Dé Danann "Lignages de la déesse Dana ", les dieux gallois sont appelés Plant Dôn "Enfants de Dôn ", et les deux théonymes sont évidemment identiques. Sur Taliesin fils de Dôn et sa mère: C. Sterckx, Taranis, Sucellos et quelques autres, Bruxelles, 2005, p. 121-134. 
réduit dans le Hanes Taliesin à un détail apparemment mineur : le poison qui constitue le reste de la mixture de Cyrridwen après l'éjection des trois gouttes d'omniscience fait exploser le chaudron où il bouillait et transforme en eau mortelle la rivière qu'il rejoint, tout comme l'éruption de la Seaghais crée une rivière fatale pour Eithne Bóinn :

"Le chaudron se brisa lorsque les trois précieuses gouttes s'en échappèrent car, hormis ces trois gouttes, le liquide était un poison qui fit périr les chevaux de Gwyddno Garanhir lorsqu'il se répandit dans la rivière en contrebas $^{127}$."

Le thème de l'eschatologie diluvienne - l'ennoiement d'un microcosme valant fin du monde - par éruption de la source cosmique n'est cependant pas bien loin de l'histoire de Gwion-Taliesin : Gwyddno Garanhir, le maître des chevaux tués par la rivière empoisonnée et le propriétaire aussi de la senne dans laquelle le petit Taliesin est pêché comme un saumon, est en effet une figure fameuse du légendaire gallois : il est le roi du royaume englouti $^{128}$.

La légende de son engloutissement est ancienne puisque sa première évocation se trouve déjà dans le fameux Livre Noir de Carmarthen :

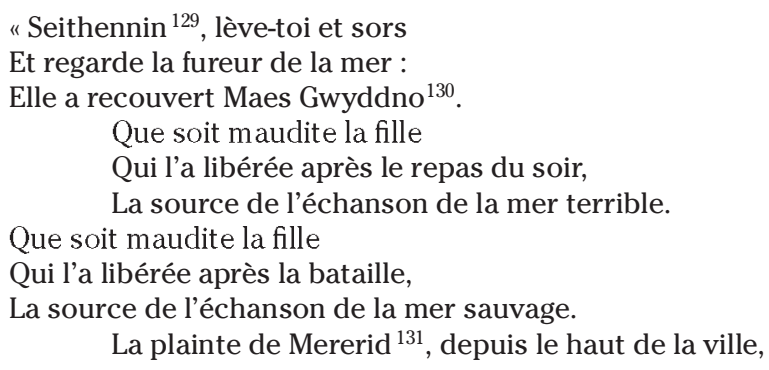

127. Hanes Taliesin in P. K. Ford, "A Fragment of the Hanes Taliesin by Llewelyn Siôn", art. cit., p. 454. Pour une tentative d'identification de cette rivière: F.J. North, Sunken Cities, Cardiff, 1957, p. 177-178.

128. Tegid Moel l'époux de Cyrridwen, semble être lui aussi le roi d'un royaume englouti qui serait devenu le Llyn Tegid " le lac de Tegid ", l'actuel lac Bala, à la suite de l'éruption d'une source dont le gardien négligent aurait un soir oublié de replacer la bonde (R. Gwyndaf, Chwedlau gwerin Cymru, op. cit., p. 51; P. C. Bartrum, A Welsh Classical Dictionary, op. cit., p. 603-604).

129. Ce nom, dérivé d'un latin Septentinus, désigne clairement ici l'un des responsables de la catastrophe mais rien ne transpire de la nature de sa faute, si ce n'est qu'il est dit d'esprit faible (synhuit vann) à la fin du poème. L'idée qu'il s'agissait du gardien des digues et qu'il s'était enivré n'apparaît que tardivement d'après les textes très suspects d'E. Williams "Iolo Morganwg": P. C. Bartrum, A Welsh Classical Dictionary, op. cit., p. 346-348.

130. "Le Pays de Gwyddno " : le nom du royaume avant qu'il ne soit englouti et appelé Cantre'r Gwaelod "le Canton au Fond [de la mer] ".

131. Mererid serait le nom de la fille coupable de la catastrophe. Son nom, forme galloise du prénom Marguerite "Perle ", pourrait peut-être n'être qu'une lectio facilior pour un plus ancien mereddig "stupide ": (J. Loth, " La légende de Maes Gwyddneu dans le Livre Noir de Carmarthen ", Revue Celtique, XXIV, 1903, p. 354; H. Le Bihan, « Beuziñ Maez 
Monte jusqu'à Dieu.

Il est normal qu'une longue expiation paie la débauche.

Ce jour, la plainte de Mererid, depuis le haut de la ville,

Monte jusqu'à Dieu.

Il est normal qu'on se repente après la débauche.

La plainte de Mererid m'épouvante ce soir

Et je ne peux guère me réjouir.

Il est normal qu'après la gloire vienne la chute.

La plainte de Mererid s'élève [du dos] d'un fort [cheval] bai ${ }^{132}$.

C'est Dieu le miséricordieux qui a amené ce [châtiment].

Il est normal que l'excès soit suivi du manque.

La plainte de Mererid me fait sortir

De chez moi.

Il est normal qu'après la gloire on meure en exil.

La tombe de Seithennin ${ }^{133}$ à l'esprit faible

Se trouve entre Caer Genedr et le rivage,

De la mer. Il fut un chef glorieux ${ }^{134}$."

Que Gwyddno ait été le roi du pays avant son engloutissement est confirmé par plusieurs allusions dans la vieille poésie - ainsi Guto’r Glyn rappelle

" La lamentation de Gwyddno Garanhir

Quand Dieu fit rouler la mer sur sa terre ${ }^{135}-$, "

et à partir du seizième siècle il apparaît que la légende est bien connue et bien située, le plus souvent au large de la péninsule de Lleyn :

"A whole cantred or hundred called Cantre'r Gwaelod, stretching itself west and south about 12 miles in length [...] hath been overwhelmed by the sea and drowned, and still a great stone wall, made as a fence against the sea, may be clearly seen [...] and is called Sarn Badric ${ }^{136}$."

Les versions populaires qui sont relevées à partir du dix-septième siècle donnent comme causes de l'ennoiement les perversions vicieuses organisées par Mererid et le coma éthylique de Seithennin, incapable de fermer les digues devant la marée montante.

Gouesnoù ", Hor Yezh 223, 2000, p. 13). Il n'est pas impossible non plus qu'il fasse allusion à une métamorphose en sirène après la submersion du pays : cf. infra.

132. Sur le sens de ce vers, généralement compris comme «la plainte de Mererid à cause des vins forts ", d'où l'idée d'une débauche d'ivrognerie : R. Bromwich, " Cantre'r Gwaelod and Ker-Is ", in C. Fox, B. Dickins (ed.), The Early Cultures of North-West Europe, Cambridge, 1950, p. 223.

133. Cette dernière strophe fait partie des Englynion y beddau (6 in A. O. H. Jarman, E. D. Jones, Llyfr Du Caerfyrddin, Cardiff, 1982, p. 36; cf. T. Jones, " The Black Book of Carmarthen "Stanzas of the Grave" ", Proceedings of the British Academy, LIII, 1967, p. 118-120).

134. Boddi Maes Gwyddno in A. O. H. Jarman, E. D. Jones, Llyfr Du Caerfyrddin, op. cit., p. 80-81. Pour la traduction, cf. R. Bromwich, " Cantre'r Gwaelod and Ker-Is. Fox », art. cit. ; L. Fleuriot, « Le thème de la ville engloutie ", in L. Fleuriot et al., Récits et poèmes celtiques, Paris, 1981, p. 234; H. Le Bihan, "Beuziñ Maez Gouesnoù ", art. cit.

135. I. Williams, Ll. Wiliams, Gwaith Guto'r Glyn, Cardiff, 1939, p. 31.

136. Robert Vaughn, cité par F. J. North, Sunken Cities, op. cit., p.153-154. 
Deux fragments mythologiques laissent néanmoins entrevoir des traits moins anecdotiques.

Le premier est l'évocation d'un duel perdu par Gwyddno face à l'un des rois des dieux, Gwynn ab Nudd, qui l'aurait épargné ${ }^{137} \ldots$ or le duel des dieux entre le représentant de la lumière et de la vie (Gwynn ab Nudd, Pwyll, Lleu...) et celui de l'obscurité (Gwyddno, Gwythyr ab Greidiol, l'adversaire anonyme d'Arawn, le voleur anonyme vaincu par Lleu...) aux calendes de mai, se terminant ainsi par la victoire de premier ou par un match nul qui conserve l'alternance nuit et jour, hiver et été, mort et vie... jusqu'à la fin des temps est un mythème récurrent ${ }^{138}$. La défaite de Gwyddno le réduirait donc à un règne dans l'Autre Monde.

Le second attribue à Gwyddno la propriété de l'un des treize trésors de la Grande-Bretagne : un récipient alimentaire inexhaustible, c'est-à-dire l'un des attributs majeurs du roi de l'Autre Monde ${ }^{139}$ :

" la manne de Gwyddno Garanhir : si on y met la nourriture d'un seul homme, il s'y trouve de quoi en nourrir cent quand on la rouvre ${ }^{140}$;"

" la manne de Gwyddno Garanhir : même si le monde entier passait devant par groupes de trois fois neuf hommes, chacun y trouverait à volonté toute la nourriture qu'il voudrait ${ }^{141}$."

\section{Fins de mondes}

La submersion du royaume serait donc à comprendre comme une eschatologie microcosmique le renvoyant à l'Autre Monde : de fait, le fond de l'océan constitue l'une des situations traditionnelles de l'Autre monde, tout comme l'horizon ou le dessous de la Terre, toutes localisations " réelles " et concevables mais totalement inaccessibles aux vivants ${ }^{142}$.

137. Ymddiddan rhwng Gwyddno Garanhir a Gwyn ab Nudd in A. O. H. Jarman, E. D. Jones, Llyfr Du Caerfyrddin, op. cit., p. 71-73. Sur Gwynn ab Nudd : B. F. Roberts, "Gwynn ab Nudd ", Llên Cymru, XIII, 1980-1981, p. 283-289; P. C. Bartrum, A Welsh Classical Dictionary, op. cit., p. 351-353.

138. Cf. N. Stalmans, Les affrontements des calendes d'été dans les légendes celtiques, Bruxelles, 1995.

139. Il ne fait guère de doute que cette corbeille inexhaustible de Gwyddno se confond avec sa senne merveilleuse qui, " une fois par an " - en fait lors d'une césure temporelle qui vaut pour le non-temps de l'Autre Monde - offre pareillement une surabondance merveilleuse. Manne et senne rejoignent ainsi le chaudron du dieu « jupitérien " (Irlandais Eochaidh Ollathair, Gaulois Sucellos... : cf. C. Sterckx, Taranis, Sucellos et quelques autres, op. cit.) qui bout inexhaustiblement le banquet de l'Autre Monde. Remarquablement, la senne de Gwyddno ramène sa pêche miraculeuse - et aussi le petit Taliesin et son omniscience! - du bouillonnement créé par le reflux dans l'embouchure de la Conwy, équivalent vénédotien de la Serven démétienne.

140. Tri thlws ar ddeg Ynys Prydain 2 in R. Bromwich, Trioedd Ynys Prydein, op. cit., p. 258.

141. Mal y cafas Culhwch Olwen in R. Bromwich, D. S. Evans, Culhwch ac Olwen, op. cit., p. 23. Nous suivons la traduction de P.-Y. Lambert, Les quatre branches du Mabinogi et autres contes gallois du Moyen Âge, op. cit., p. 144, si ce n'est que nous rendons le mot mwys par " manne " et non pas par "plat".

142. Cf. D. E. Edel, "Antipoden, ankers en een wereld-onder-het-water ", in A. M. J. Van Buuren et al., Tussentijds. Bundel studies aangeboden aan W. P. Gerritsen ter gelegenheid 
Il existe cent autres versions de ce mythème d'une inondation cataclysmique ennoyant un microcosme, le plus souvent à la suite d'une éruption de la source cosmique.

- Au Pays de Galles, une triade conservée, en latin, par la Cronica de Wallia de treizième siècle, énumère ainsi

"Les royaumes que la mer a détruits.

Celui de Teithi Hen ab Gwynnan ${ }^{143}$, roi de Caerrhihog qui est appelée depuis lors Ynys Teithi Hen et qui se trouvait entre Anglesey et l'Irlande. Aucun homme ni aucun animal domestique n'échappa, sauf Teithi Hen luimême sur son cheval. Et après cela, il resta transi de frayeurs pour tout le reste de sa vie.

Le deuxième royaume est celui d'Helig ab Glannog ${ }^{144}$. Il se trouvait entre Cardigan et Bardsey et s'étendait jusqu'à Saint David. Cette terre était bonne, fertile et plaine, et elle s'appelait Maes Maichgen. Elle allait de l'estuaire jusqu'à Lleyn et jusqu'à Aberdovey.

La mer a détruit un troisième royaume : le royaume de Rhedfoe ${ }^{145} \mathrm{ab}$ Rheged ${ }^{146}$

Et Robin Gwyndaf évoque aussi la légende du lac de Llangors qui aurait noyé la méchante princesse Syfaddan et son royaume, dans lequel chantent encore ses oiseaux merveilleux qui ressemblent fort aux oiseaux de l'Autre Monde traditionnellement attribués à la déesse Rhiannon ${ }^{147}$.

- Le folklore irlandais connaît de nombreux contes d'inondations catastrophiques dues à la négligence dans le devoir de mettre une bonde à une source merveilleuse ${ }^{148}$. L'un d'eux en particulier repose sur des traditions anciennes et mérite ici d'être évoqué.

Il est connu à travers plusieurs versions que la dernière, l'Oidheadh Eachdach mhic Maireadha, rassemble en une synthèse artificielle mais commode lorsque, comme ici, le propos n'est pas d'en disséquer l'élaboration. En voici le résumé.

Eochaidh mac Maireadha, fils d'un roi de Munster, tombe amoureux de sa belle-mère, la reine Eibhliu, déesse issue du Brugh na Bóinne. Son frère Ríbh lui conseille de l'enlever pour ne pas dépérir d'amour. Les deux frères s'enfuient avec Eibhliu et mille suivants, puis ils se séparent. Ríbh arrive sur

van zijn vijftigste verjaardag, Utrecht, 1985, p. 101-114 et 339-342; G. Hily, L'Autre Monde ou la source de vie, Bruxelles, 2003.

143. Cf. P. C. Bartrum, A Welsh Classical Dictionary, op. cit., p. 608.

144. Helig ab Glannog est connu comme le père de plusieurs saints et l'ancêtre de deux clans de Vénédotie. Sa légende semble n'être qu'une autre version de celle du Cantre'r Gwaelod (F. J. North, Sunken Cities, op. cit. ; P. C. Bartrum, A Welsh Classical Dictionary, op. cit., p. 362-363).

145. Rhedfoe est totalement inconnu par ailleurs : P. C. Bartrum, A Welsh Classical Dictionary, op. cit., p. 552.

146. T. Jones, "Triawdd lladin ar y gorlifiadau ", Bulletin of the Board of Celtic Studies, XII, 1947-1949, p. 79-83.

147. R. Gwyndaf, Chwedlau gwerin Cymru, op. cit., p. 73.

148. Voir par exemple D. Hyde, «Editorial Note », Béaloideas, II, 1930, p. 252. 
le domaine du dieu Midhir qui tue tous ses chevaux, puis lui donne l'un des siens capable de porter à lui tout seul tous les bagages de la compagnie. Quelques jours plus tard, le cheval se couche et, quand il se relève, une source jaillit, inonde toute la contrée, noie Ríbh et ses compagnons sous un lac connu depuis comme le Lough Ríbh ${ }^{149}$. De leur côté, Eochaidh et Eibhliu arrivent au domaine du dieu Aonghus : autrement dit au Brugh na Bóinne dont est précisément originaire Eibhliu. Aonghus leur ordonne de partir et tue aussi tous leurs chevaux. Quand il renouvelle son ordre de départ et qu'ils se déclarent incapables d'obéir sans leurs bêtes, il leur donne un cheval qui peut porter tous leurs bagages à lui tout seul, mais en leur enjoignant de ne pas le laisser s'arrêter en route ${ }^{150}$. Arrivés à en Ulster, ils déchargent les bagages mais lorsque le cheval se relève ${ }^{151}$, une source jaillit. Eochaidh l'entoure d'une margelle, la bouche d'une bonde et commet une gardienne à son service. Un jour, la gardienne oublie de remettre la bonde en place : la source déborde, inonde la région et noie Eochaidh et tous les siens, à l'exception d'une fille, Líban, et de son petit chien. Et cette inondation devient le fameux Lough Neagh. Líban reste un an sous la mer, après quoi elle est métamorphosée en saumon à la voix de sirène et son petit chien en loutre. Trois cents ans plus tard, ils sont tous deux pêchés dans un filet et mis dans une jarre en guise d'aquarium. Le choix est alors laissé à Líban : rester encore trois cents ans dans son état ou être baptisée, mourir aussitôt et gagner le paradis. Comme l'exige l'époque chrétienne à laquelle la légende est ainsi contée, elle choisit la seconde option et devient sainte Fainche Muirghein ${ }^{152}$.

Remarquablement, le nom de Fainche Muirghein signifie "Corvidé Femelle Née de la Mer " et semble rapprocher Líban de la déesse Badhbh, l'une des formes de la Mórrigháin, quant au détail de son inséparable petit chien, il évoque vraisemblablement l'antique déesse Epona et son avatar gallois Rhiannon ${ }^{153} \ldots$

- Le thème des pierres bondes dont l'enlèvement entraînerait l'inondation du monde, ou du moins d'un canton-microcosme, est par ailleurs très général. Divers relevés en ont été faits, notamment pour la France ${ }^{154}$.

149. Cette partie de la légende n'est clairement qu'un doublet attachant au Lough Ríbh le même scénario qu'au Lough Neagh : E. MacNéill, " The Mythology of Lough Neagh ", Béaloideas, II, 1929, p. 117; E. J. Gwynn, The Metrical Dindsenchas, op. cit., IV, p. 389-390. 150. Le Dinnsheanchas métrrique (E. J. Gwynn, The Metrical Dindsenchas, op. cit., IV, p. 66) précise qu'ils ne peuvent pas laisser pisser le cheval sous peine de mort.

151. Selon le Dinnsheanchas métrique, il pisse et son urine creuse le sol pour faire jaillir la source.

152. O. Bergin, R. I. Best, Lebor na hUidre, Dublin, 1929, p. 95-100. Sur cette synthèse, voir E. MacNéill, "The Mythology of Lough Neagh ", art. cit., et cf. O. Bergin, "Observations on The Mythology of Lough Neagh ", Béaloideas, III, 1930, p. 246-252; D. Ó hÓgáin, Myth, Legend and Romance, op. cit., p. 181 et 271.

153. Cf. C. Sterckx, Eléments de cosmogonie celtique, Bruxelles, 1986, p. 9-47; D. Ó Corráin, F. Maguire, Irish Names, Dublin, 1990, p. 107-108

154. Y. Vadé, "Sur la maternité du chêne et de la pierre ", Revue de l'Histoire des Religions, CXCIV, 1977, p. 3-41 ; J.-P. Lelu, « La Pierre Couvretière d'Ancenis et les "pierres bondes" ", 
On note toutefois dans ces derniers que la part de l'Armorique y est remarquablement grande ${ }^{155}$. Ainsi à Combourg (Ille-et-Vilaine), la pierre bonde au fond de la fontaine Margatte empêche celle-ci d'inonder tout le pays ${ }^{156}$; à Dol-de-Bretagne (Ille-et-Vilaine), si on ôte la Pierre Buquet, le pays sera submergé ${ }^{157}$; à Saint-Samson (Côtes-d'Armor), la pierre de la Tremblaye est un menhir tenu pour l'une des trois clefs de la mer : l'enlèverait-on que la mer recouvrirait " toute la France ${ }^{158}$ "; à Trans (Ille-et-Vilaine) l'enlèvement d'une pierre bonde au milieu du bois de la Villecartier noierait tout le pays ${ }^{159}$; au Vieux-Viel (Ille-et-Vilaine), une pierre bonde bouche l'entrée de l'Abîme et empêche la mer de déferler ${ }^{160}$... Mieux encore, la source primordiale de toutes les eaux vives a été identifiée, jusqu'au début du siècle dernier, à la source du Blavet, ou plutôt, comme l'est régulièrement l'Autre Monde des Celtes, sous l'océan ou en dessous de la Terre même : l'Eil de la Mer, dont le Blavet n'aurait été que l'un des écoulements ${ }^{161}$, et sans doute est-ce aussi le souvenir d'une source merveilleuse, hantée par un poisson primordial, susceptible de s'enfler catastrophiquement au pied d'un arbre dont les fruits y tombent, que l'on retrouve dans la légende de Saint-Potan (Côtes-d'Armor) : là, la source coule au pied d'un chêne, sert de demeure à une anguille-fée et elle ennoierait tout le pays si l'on s'avisait de déraciner le chêne ${ }^{162}$.

La plus célèbre légende d'ennoiement eschatologique d'un microcosme y est, bien sûr, celle de la ville d'Is, censée se trouver aujourd'hui sous la mer au large de Douarnenez (Finistère) ${ }^{163}$.

Bulletin de la Société de Mythologie Française, 212, 2003-2004, p. 7-9. On doit aussi rappeler le thème du royaume englouti (Lyonesse) dans les légendes arthuriennes : cf. P. Fowler, C. Thomas, «Lyonesse Revisited », Antiquity, LIII, 1979, p. 175-189.

155. En dehors de la France, c'est en Galice - autre région aux souvenirs celtes remarquablement nombreux et significatifs - que le motif apparaît le plus fréquent et le plus proche : cf. L. Monteagudo García, Galicia legendaria y arqueológica : problema de las " ciudadas asolagadas ", Madrid, 1957.

156. J. Gastard, Combourg, Combourg, 1962, p. 8-9.

157. J.-P. Clébert, « La France engloutie ", in A. Michel, J.-P. Clébert, Histoire et guide de la France secrète, Paris, 1968, p. 263.

158. L. de Villers ["Lucie de V. H. »], " Le menhir de Saint-Samson ", Revue des Traditions Populaires, XVII, 1902, p. 353.

159. A. Dagnet, Les bords du Couesnon, Fougères, 1906, p. 42 n.2

160. G. Guénin, Le légendaire préhistorique de la Bretagne, Rennes, 1995, p. 235.

161. P. Sébillot, Le folklore de la France, Paris, 1904-1907, II, p. 324; F. Cadic, Contes et légendes de Bretagne, Paris, 1914, p. 3-4.

162. E. Nourry ["Paul Saintyves"], Corpus du folklore des eaux en France et dans les colonies françaises, Paris, 1934, II, p. 118; C. Sterckx, Les dieux protéens des Celtes et des Indo-Européens, op. cit., p. 12.

163. Cf. essentiellement (et avec bibliographie exhaustive) P. Galliou et al., La légende de la ville d'Ys, Quimper, 2002 et J. Hascoët, "À la recherche de Ker Is, la ville de l'Autre Monde ", 2007 (à paraître). Des légendes analogues sont contées ailleurs en Armorique : à Cancale (Ille-et-Vilaine), à Erquy (Côtes-d'Armor)... : M. Déceneux, Bretagne celtique, Brest, 2002, p. 50-53. 
Son existence paraît déjà attestée au neuvième siècle par une remarque d'Abbon de Saint-Germain-des-Prés :

"Lutèce : ainsi te nommait-on autrefois mais à présent ton nom vient de la ville d'Isia sise au centre du vaste pays des Grecs, brillante par son port magnifique entre tous : de cette Isia que hante l'avide Argien, tout assoiffé de trésors. Un nom bâtard composé par une sorte de métaplasme pour toi, sa rivale, dépeint bien ton aspect, ô Lutèce. Ce nom nouveau que le monde te donne, c'est Paris, c'est-à-dire "Pareille à Isia" : avec raison car elle t'est semblable ${ }^{164}$."

En effet, l'étymologie populaire traduisant le nom de Paris par "Semblable à l'Is " cornouaillaise est constante, attestée dès la première mention de la légende ${ }^{165}$ jusqu'à la tradition populaire aujourd'hui ${ }^{166}$.

Cette plus ancienne version, dans les Croniques et ystoires des Bretons de Pierre Le Baud, est encore sommaire :

La grande cité de Ys situee pres la grand mer, si comme on dit, fut en celuy temps, pour les pechez des habitants, submergee par les eaux issant de celle mer qui trespasserent leurs termes; laquelle submersion le Roy Grallons, qui lors estoit en celle cite, eschappa miraculeusement : c'est à savoir par le merite de sainct Guingalreus, duquel il est touche cy-apres. Et dit l'on que encores en appierent ses vestiges sus la rive de celle mer, qui de l'ancien nom de la cite est jusques a maintenant appelee $\mathrm{Ys}^{167}$.

D'autres détails apparaissent par la suite et la version canonique est enfin donnée en 1636 par Albert Le Grand et Dubuisson-Auybenay :

" [Gwennolé] alloit souvent voir le Roy Grallon en la superbe cité d'Is, \& preschoit fort hautement contre les abominations qui se commettoient en cette grande Ville toute absorbee en luxes, debauches et vanitez, mais demeurans obstinez en leurs pechez. Dieu revela à S. Guennole la juste punition qu'il en vouloit faire : Saint Guennole estant allé voir le Roy, comme il avoit de coustume, discourans ensemble, Dieu lui revela l'heure du chastiment exemplaire des Habitans de cette Ville estre venuë. Le Saint, retournant comme d'un ravissement \& extaze, dit au Roy : "Ha! Sire, Sire! Sortons au plustost de ce lieu : car l'ire de Dieu le va presentement accabler : Vostre Majeste sçait les dissolutions de ce peuple, on a beau le prescher, la mesure est comble, faut qu'il soit puny: hastons nous de sortir, autrement nous serons accueillis et enveloppez en ce

164. Abbon de Saint-Germain-des-Prés, Bella parisiaca in H. Waquet, Abbon. Le siège de Paris par les Normands, Paris, 1964, p. 12-13.

165. Pierre Le Baud écrit vers 1500 : Les Corisopitenses se vantent le dit nom de Paris lui avoir été attribué comme " Pareille à Is " (C. de la Lande de Calan, Croniques et ystoires des Bretons par Pierre Le Baud, Nantes, 1907-1922, III, p. 21).

166. J. Hascoët, "La longue vie de l'étymologie Ker-Is, Par-is ", Britannia Monastica X, 2006, p. 90, cite ainsi le témoignage ingénu de Jean-Marie Déguinet vers 1900 : « car pour les vieux Bretons, le mot Paris veut dire simplement "Pareil à Is", la syllabe par signifiant exactement "pareil"..."

167. C. de la Lande de Calan, Croniques et ystoires des Bretons par Pierre Le Baud, op. cit., III, p. 21. Sur la figure de Grallon : B. Tanguy, "Grallon, roi de Cornouaille ", $A r$ Men, 14, 1988, p. 30-44, "Gradlon ", in P. Galliou et al., La légende de la ville d'Ys, op. cit. J. Quaghebeur, La Cornouaille du IX $X^{e}$ au XII siècle, Rennes, 2002, p. 39-50. 
même malheur." Le Roy fit incontinent trousser bagage \&, ayant fait mettre hors ce qu'il avoit de plus cher, monte à cheval avec ses Officiers \& domestiques, \& a pointe d'eperon se sauve hors de la ville. À peine eust-il sorti les portes qu'un orage violent s'eleva avec des vents si imperieux que la Mer, se jetant hors de ses limites ordinaires et se jetant de furie sur cette miserable Cite, la couvrit en moins de rien, noyans plusieurs milliers de personnes, dont on attribua la cause principale a le princesse Dahut ${ }^{168}$, fille impudique du bon Roy, laquelle perit en cet abysme \& cuida causer la perte du Roy en un endroit qui retient le nom de Toul-Dahut ou Toul-Alchuez, c'est-à-dire le Pertuis Dahut ou le Pertuis de la Clef, pour ce que l'histoire porte qu'elle avoit pris à son Père la Clef qu'il portoit pendante au col, comme symbolle de la Royauté. Le Roy s'estant sauve d'heure, alla loger a Land-Tevenec avec s. Gunennolé, lequel il remercia de cette délivrance, puis se retira a Kemper ${ }^{169}$."

" En ce lieu, la tradition et vieilles histoires de Bretagne portent que fut la ville d'Ys ou Yns (Belon, en ses Observations, dit que Paris est appelée quod sit par Ys, égal en grandeur à la ville d'Ys qu'il prend pour Le Caire), abismée de temps d'un roy Grallon par la meschanceté de sa fille, laquelle, par commandement d'une voix céleste, il jeta de dessus son cheval et abandonna en la mer qui gagnoit et le suivoit, luy fuyant à cheval et se sauvant comme Loth de Sodome ${ }^{170}$."

Ces détails sont apparemment reçus de la tradition populaire car Noël Mars, le très catholique abbé de Landévennec, les condamne sous ce prétexte :

"Ce qui me fait réfuter ceste histoire comme apocrife c'est que les contes qu'en font les bonnes femmes de la basse Bretagne... sont tout à fait ridicules ${ }^{171}$."

Les analogies entre la légende galloise du Cantre'r Gwaelod et celle-ci sont évidentes : la ville dissolue, la colère divine, l'ouverture des digues due à l'ivresse du responsable, la fuite à cheval devant le flot meurtrier, le roi qui perd tout sauf la vie...

On devine sans peine qu'il a dû y avoir, à assez haute époque pour que Gallois et Bretons en gardent ainsi conjointement l'héritage, l'adaptation par un clerc de la légende biblique sur la destruction de Gomorrhe et de Sodome à une tradition indigène d'eschatologie par submersion causée par une approche indue ${ }^{172}$ de la source cosmique, bouche de toutes les eaux.

168. Dans les versions tardives, la fille de Grallon est appelée Ahès et il faut noter que, selon la tradition orale, elle est métamorphosée en sirène, comme Líban après la submersion d'Is (A. Le Braz, La légende de la Mort chez les Bretons armoricains, Rennes, rééd. 1994, p. 246) : or l'un des noms coutumier de la sirène armoricaine est March'arid ar Gwall Amzer " Marguerite du Mauvais Temps ", soit une appellation homonyme de celle de la coupable de la submersion du Cantre'r Gwaelod gallois (J.-P. Piriou, " Quelques remarques à propos de l'ancien mystère de saint Gwennolé ", in G. Le Menn, J.-Y. Le Moing [éd.], Bretagne et pays celtiques. Mélanges offerts à la mémoire de Léon Fleuriot [1923-1987], op. cit., p. 204-205).

169. A. Le Grand, Vies des saints de la Bretagne armorique, 1837, p. 56-57.

170. F.-N. Dubuisson-Aubenay, Itinéraire de Bretagne, Nantes, 2000, I, p. 217.

171. Noël Mars, Histoire du royal monastère de S. Guennolé de Landévennec (1648), cité par J. Hascoët, " À la recherche de Ker Is... ", 2007 (à paraître)

172. Fréquemment imputée à une femme : Eithne Bóinn, Mererid, Dahud-Ahès-Marc'harid ou quelque autre. L'analyse de H. Amemiya ("La déesse de la mer bretonne", in 
Soit qu'ils aient déjà figuré dans la forme première, perdue, de cette pieuse adaptation, soit qu'ils aient été greffés secondairement à l'une des étapes de la transmission orale, d'autres détails remarquables, issus de très anciens concepts celtes sur le monde et son destin, se découvrent dans un conte armoricain recueilli au dix-neuvième siècle mais malheureusement fort oublié - et non traduit - depuis sa publication en 1870 : Kristof ${ }^{173}$.

Kristof laisse la vie sauve à un petit poisson qu'il a capturé dans la baie de Douarnenez, en échange d'une promesse de réaliser tous ses souhaits. Il se rend ensuite à Is, sous laquelle il y a

«Un chêne immense au milieu de la mer, vieux d'on ne sait combien de siècles. Personne n'a jamais pu l'atteindre et on n'en connaît même plus le nom. Les savants disent qu'il existait depuis le début du monde et servait de fondement à la ville d'Is. "

Il demande alors :

"Que par la vertu de mon petit poisson cet arbre soit déraciné [du fond] de la mer... et que je puisse le monter comme un cheval! »

Il parcourt ainsi les rues d'Is. Ahès, la fille du roi Grallon se moque de son équipage et Kristof émet un nouveau souhait :

«Par la vertu de mon petit poisson, puisque vous vous gaussez, je voudrais que vous soyez enceinte à l'instant! ”

La grossesse de la princesse devient évidente après quelques mois et cause un grand scandale, d'autant plus qu'elle affirme ne pas savoir comment cela lui est arrivé. Elle accouche enfin d'un garçonnet et un vieux druide, sorti des bois et qui y disparaît à nouveau aussitôt après, enseigne à Grallon comment découvrir l'identité du père : le roi doit suspendre sa couronne à un fil tendu entre deux piquets; celui sur la tête de qui elle choira sera le géniteur. Kristof est ainsi dénoncé comme tel quoique Ahès jure n'avoir jamais copulé avec lui, ni avec qui que ce soit d'ailleurs... Grallon les marie pourtant mais, bientôt dégoûté d'un gendre qu'il juge indigne de lui, il fait enfermer Kristof, Ahès et leur bébé dans un coffre en bois qu'il fait jeter à la mer. Kristof fait appel à son poisson et lui enjoint de les amener sur une île, de leur y bâtir un palais merveilleux et de jeter un pont entre l'île et Is. Apprenant ce qu'il en est, Grallon envoie ses soldats pour s'emparer de Kristof mais ils sont tous tués, sauf un seul... Après d'autres passes d'armes, Grallon finit par apprécier son gendre et lui propose de revenir à Is. À ce moment, Kristof l'avertit :

F. Favereau, H. le Bihan (éd.), Littératures de Bretagne. Mélanges offerts à Yann-Ber Piriou, Rennes, 2006, p. 253-266) reste très littéraire.

173. Hervé Le Bihan en promet une étude en breton. Il en a déjà donné une version normalisée, avec quelques brefs commentaires, dans sa thèse d'habilitation inédite Eus Ledav da Vreizh, Université Rennes 2, 2005, p. 39-46. L'étude de M. Boyd, « L'enfant d'Ahez ou le fabuleux destin du conte Kristof, une légende de la ville d'Is ", in F. Favereau, H. le Bihan (éd.), op. cit., p. 309-326 s'intéresse surtout au destin de cette légende dans la littérature contemporaine. 
"Votre ville d'Is ne durera plus guère maintenant car j'ai déraciné le chêne qui empêchait la mer de la submerger à la première grande marée. N'oubliez pas ce que je vous dis, ô roi : la ville d'Is sera entièrement recouverte par la mer."

Ce qui ne manque pas d'arriver ${ }^{174}$.

Le grand chêne qui sert d'assise à Is est évidemment l'arbre cosmique qui soutient le monde, ici microcosmisé en un petit royaume armoricain. Son " déracinement " apparaît comme un étonnant souvenir des plus anciennes conceptions eschatologiques des Celtes, selon lesquelles, nous l'avons vu, la fin du monde est causée par un écroulement de l'axis mundi, la chute du ciel sur la terre et " sur la tête des hommes ", et une submersion générale renvoyant au principe.

Et l'ensemble du dossier découvre enfin le sens de l'un des exploits prêtés à Nynnio et à Peibio lorsqu'ils arrivent à tirer du Llyn Lliwan, la source cosmique des Gallois ${ }^{175}$, le monstre responsable de l'inondation du microcosme britannique causée par l'éruption de cette source : ils mettent fin à une césure interéonique et assurent donc la (re)naissance du monde pour un nouvel éon ${ }^{176}$.

\section{L'œil et l'eau}

Le détail que l'un d'eux - Peibio? - perd un œil dans une aventure évidemment analogue - celle du Llyn yf Afanc - semble être également significative $^{177}$.

174. A. Troude, G. Milin, Ar marvailler brezounek, Brest, 1870, p. 302-339

175. Cf. supra.

176. Le lien entre les bœufs et une source cosmique distribuant alternativement la vie et la mort se retrouve peut-être dans la notice topologique irlandaise sur le Slieve Gamph, en Connaught : une source qui y sourd est comptée comme l'une des merveilles de l'Irlande car, depuis qu'y aurait été jetée la tête coupée d'un certain Gamh Gruadhsholas, elle donne une eau alternativement grise et saumâtre - morte et mortifère donc - et claire et douce - vive et vivifiante donc - (Dinnsheanchas métrique = E. J. Gwynn, The Metrical Dindsenchas, op. cit., III, p. 436; Dinnsheanchas de Rennes $137=$ W. Stokes, The Prose Tales in the Rennes Dindsenchas, art. cit., XVI, p. 145). Or la désignation traditionnelle de la région est Sliabh Dhamh en irlandais, Ox Mountains en anglais " les monts des Bœufs ", ce qu'on estime généralement être une erreur causée par l'homophonie de Sliabh Gamh " Mont de Gamh " et Sliabh Dhamh « Mont des Bœufs " (J. O’Donovan, Annals of the Kingdom of Ireland by the Four Masters, Dublin, 1856, p. 1286)... mais la tradition populaire n'a-t-elle pas ici raison contre la topologie " savante " du Dinnsheanchas? Un cas analogue est attesté en Armorique pour la rivière d'Etel, dont la célèbre " barre " s'apparente en tout point au mascaret de la Severn : tout comme cette dernière est associée au Llyn Lliwan, l'armoricaine l'est à trois yeux d'eau sans fond qui se gonflent à gros bouillons à chaque marée - autre microcosme temporel - et qui offrent l'une un eau saumâtre et mortifère, la deuxième une eau mêlée, la troisième une eau vive et vivifiante : L. Maître - P. de Berthou, Cartulaire de l'abbaye de Sainte-Croix de Quimperlé, Paris, 1896, p. 219; cf. B. Tanguy, «Marais, étangs et zones humides dans la nomenclature toponymique en Bretagne ", Kreiz, VIII, 1998, p. 35.

177. De même, la bave qui déborde incessamment de la bouche de Peibio pourrait bien être une transposition de l'éruption irrépressible de la source cosmique. 
En effet, dans les langues et les traditions celtiques le terme « œil » désigne non seulement l'organe physique mais aussi une source et il est vraisemblable que les conceptions anciennes sur le mécanisme de la vision, conçue comme issue d'un feu résidant dans "l'eau " (l'humeur vitrée) de l'œeil, a joué sur l'identification spécifique de la source cosmique à un œil $^{178}$ : en témoignent notamment dans l'Antiquité la forme prêtée au bassin sacré de Bibracte, à Glux (Nièvre) ${ }^{179}$ et le patronage de la déesse Sulis sur les sources sacrées de Bath (Somerset) ${ }^{180}$, plus tard l'appellation Eil de la Mer attribuée à la source cosmique par la légende armoricaine du Blavet ou l'assimilation du Llyn Llygad Ych à l'œil de Peibio ${ }^{181}$.

\title{
La dispute primordiale
}

Ce n'est pas tout. Le thème des deux animaux primordiaux qui se disputent et dont l'un des deux perd un œil dans une eau qui est évidemment aussi primordiale est clairement attesté en Irlande dans un mythe dont tout indique qu'il a été pancelte.

- Cette fois, il ne s'agit pas de deux bovidés mais d'un saumon et d'un aigle.

Fionntan mac Bóchra, déjà cité, est en effet engagé dans un terrible conflit avec un aigle aussi primordial que lui. Ce conflit est évoqué dans un dialogue prêté aux deux adversaires.

\author{
" (l'aigle) Fionntan, fils de la belle Bóchra, \\ Puisque tu es poète et devin, \\ Dis-nous maintenant sans tergiverser \\ Les maux et les merveilles que tu as connus \\ $[\ldots]$. \\ (Fionntan) Après la pluie noire du Déluge, \\ Le Seigneur, à mon grand dam, \\ Me transforma en saumon. \\ [...] \\ Un corbeau ${ }^{182}$ vint de la froide Achill \\ Au-dessus de l'estuaire d'Assaroe. \\ Je ne le cache pas, quoique ce soit un mystère,
}

178. C. Sterckx, L'œil, la main, le pied : ordalies trifonctionnelles 2007 (à paraître).

179. J. Gran Aymerich, M. Almagro Gorbea, El estanque monumental de Bibracte (Mont Beuvray, Borgoña), Madrid, 1991. Cf. B. Sergent, «Bibracte : mythologie d'un bassin, in G. Capdeville (éd.), L'eau et le feu, op. cit., p. 321-328 qui, sans connaître l'aspect oculaire du bassin, a bien mis en évidence son rapport avec la légende de Nynnio et Peibio au Llyn yr Afanc.

180. C. Sterckx, L'œil, la main, le pied : ordalies trifonctionnelles 2006 (à paraître).

181. Cf. supra.

182. La poésie irlandaise confond tous les oiseaux noirs plus ou moins nécrophages et utilise indifféremment leurs noms selon les besoins de la métrique : cf. C. Sterckx, Des dieux et des oiseaux, Bruxelles, 2000, p. 43-44. 
Il m'enleva l'un de mes yeux.

Le Borgne d'Assaroe : tel est le surnom qui me suit

Depuis cette nuit cruelle.

J'ai perdu mon œil

$[\ldots]$

(l'aigle) C'est moi qui ai gobé ton œil,

Naïf Fionntan.

Je suis l'aigle gris,

Seul au milieu d'Achill ${ }^{183}$...

- L'existence de traditions analogues en Écosse semble assurée par une légende recueillie au dix-neuvième siècle à Lochaber (Highland) qui cite comme animal primordial une truite éborgnée de la même façon ${ }^{184}$ et aussi par la représentation d'un rapace s'attaquant à un saumon sur au moins deux pierres pictes, à Latheron (Caithness) ${ }^{185}$ et à Saint Vigeans (Angus) ${ }^{186}$, d'une façon cette fois peut-être plus proche de la version galloise telle que préservée dans Mal y cafas Culhwch Olwen.

- Là encore, les deux animaux primordiaux sont un aigle et un saumon qu'il mutile :

" Il y a longtemps, dit l'aigle, que je suis venu ici. À mon arrivée, il y avait une roche du sommet de laquelle je becquetais les arbres chaque soir. Maintenant, elle n'a plus qu'une paume de haut [...]. Cependant, une fois, j'allai chercher ma nourriture au Llyn Lliwan; arrivé au plan d'eau, j'enfonçai mes serres dans un saumon, pensant qu'avec lui ma nourriture était assurée pour longtemps mais il m'entraîna dans les profondeurs et ce ne fut qu'à grand-peine que je pus me détacher de lui. Moi et ma famille, nous nous mîmes en campagne ardemment pour tâcher de le mettre en pièces mais il m'envoya des messagers pour s'arranger avec moi et il vint en personne me livrer cinquante harponnées de la chair de son $\operatorname{dos}^{187}$."

183. Ársaid sin, a eoin Accla 13, 20-22 in K. Meyer, "The Colloquy between Fintan and the Hawk of Achill ", in O. Bergin et al., Anecdota from Irish Manuscripts, op. cit., p. 27. Achill est une île au large des côtes du Connaught; Assaroe est une cascade célèbre pour l'abondance des saumons qui s'y rendent, près de l'embouchure de l'Erne.

184. K. H. Jackson, The International Popular Tale and Early Welsh Tradition, Cardiff, 1961, p. 77; C. Sterckx, Les dieux protéens des Celtes et des Indo-Européens, op. cit., p. 13. Tout comme les anciens Celtes rangeaient facilement dans une même espèce aigles, corbeaux et autres oiseaux noirs analogues, ils confondaient volontiers truites et saumons.

185. J. Anderson, « Notices of a Sculptured Stone with Ogham Inscription, from Latheron ", Proceedings of the Society of Antiquaries of Scotland, XXXVIII, 1904, p. 534-538.

186. A. Ritchie, Picts, Londres, 1989, p. 36; T. O. Clancy, «The Drosten Stone : A New Reading ", Proceedings of the Society of Antiquaries of Scotland, CXXIII, 1993, p. 345-353. Pour les invraisemblables interprétations chrétiennes qu'on a prétendu en faire : C. Sterckx, Dieux d'eau : Apollons celtes et gaulois, op. cit., p. 10 n.1; A. Ritchie, «Paganism among the Pïcts and the Conversion of Orkney ", in J. Downes, A. Ritchie (ed.), Sea Change : Orkney and Northern Europe in the Later Iron Age AD 300-800, Balgavies, 2003, p. 4.

187. Mal y cafas Culhwch Olwen in R. Bromwich, D. S. Evans, Culhwch ac Olwen, op. cit., p. 31-33. Cf. C. Sterckx, Les dieux protéens des Celtes et des Indo-Européens, op. cit., p. 11-18. 


\section{Le dialogue des animaux}

L'association des deux bœufs à un intervalle interéonique et à la naissance d'un nouvel éon trouve vraisemblablement un autre écho dans la croyance, encore très vivante récemment, que les couples de bœufs ${ }^{188}$ ont la connaissance du passé et du présent, mais aussi de l'avenir pendant la nuit de Noël ${ }^{189}$ : tenue pour celle du solstice d'hiver, celle-là constitue en effet depuis très longtemps l'une des césures traditionnelle entre ces deux microcosmes temporels que sont l'année échue et l'année écoulée ${ }^{190}$.

\section{Les deux porchers}

Le thème de l'affrontement entre deux êtres primordiaux passant par une série de métamorphoses, tantôt humaines, tantôt animales, tenait manifestement une place tellement importante dans la tradition qu'il a été repris pour donner une étiologie remontant à l'origine même du monde pour la razzia du bétail de Cooley, le mythe héroïque majeur de l'ancienne Irlande ${ }^{191}$.

Cette étiologie est l'objet du Da chophur nan ndá mhuicidhthe ${ }^{192}$ : deux dieux souverains mineurs, Foraoí Badhbh Dearg du Munster et Ochall Oichne du Connaught ${ }^{193}$, font la paix après une longue dispute mais leurs porchers respectifs ${ }^{194}$, Friuch et Rucht, se prennent à leur tour de querelle et en viennent à jeter chacun un sort funeste sur le troupeau de l'autre, ce qui leur vaut d'être démis de leur fonction; enragés, ils se battent alors sous les formes successives de deux saumons, de deux cerfs, de deux guerriers,

188. Normalement remplacés par des couples de chevaux quand ces derniers prennent la place des bœufs comme bêtes de trait : D. Giraudon, "Merveilles de la nuit de Noël ", Ollodagos, XX, p. 187.

189. Voir A. Van Gennep, Le folklore français. Paris, 1998-1999, p. 2690-2694; D. Giraudon, "Merveilles de la nuit de Noël », Ollodagos, XX, 2006, p. 178-205.

190. C'est sans doute l'une des raisons qui y ont fait situer la naissance de Jésus : ces césures impliquent en effet la rencontre et la confusion de notre monde avec l'autre, offrant la possibilité de passer de l'un à l'autre : tout comme pendant la nuit des calendes d'hiver ou durant les douze coups de minuit à Noël, les humains peuvent pénétrer dans l'Autre Monde et tenter d'en dérober les trésors, les êtres de l'Autre Monde peuvent venir dans le nôtre : ainsi les revenants pendant la nuit des calendes d'hiver ou... Dieu lui-même pendant la nuit de Noël.

191. Sur la parenté entre cette épopée héroïque et les autres grands mythes héroïques indo-européens, dont particulièrement l'Iliade grecque, voir B. Sergent, Celtes et Grecs, Paris, 1999-2004, I, p. 99-200.

192. Ed. U. Roider, De chophur in dá muccida, Innsbruck, 1979, p. 24-58. Cf. C. Sterckx, Sangliers Père \& Fils, op. cit., p. 34-36.

193. Vraisemblablement deux des souverains "provinciaux ", l'un au sud et l'autre à l'ouest, correspondants divins de deux des quatre sages primordiaux " totalisant" Fionntan (cf. supra). Sur Foraoí Badhbh Dearg et Ochnall Oichne : C. Sterckx, Des dieux et des oiseaux, op. cit., p. 30-36.

194. C'est en fait là une fonction éminente et symboliquement significative: cf. P. Ní Chatháin, "Swineherds, Seers and Druids ", Studia Celtica, XIV-XV, 1980-1981, 200-211; D. Poli, « Le divin porcher ", Études Celtiques, XXIX, 1992, p. 375-382. 
de deux spectres, de deux dragons... Ils se métamorphosent finalement en deux animalcules, l'un dans la source de Cooley en Ulster, l'autre dans une source du Connaught; ils y sont l'un et l'autre bus par des vaches et, ainsi, renaissent sous la forme de deux taureaux merveilleux Donn " le Brun " de Cooley et Finnbheannacht « le Blanc Cornu » de Connaught; ainsi incarnés, ils sont la cause de la grande guerre entre l'Ulster et le reste de l'Irlande, connue sous le nom de " razzia du bétail de Cooley ", au terme de laquelle Donn tue Finnbheannacht et meurt lui-même de l'effort que cela lui coûte ${ }^{195}$.

\section{Tableau récapitulatif}

\begin{tabular}{|c|c|c|}
\hline $\begin{array}{l}\text { 1. Deux rois, les frères } \\
\text { Nynnio et Peibio se } \\
\text { querellent }\end{array}$ & & $\begin{array}{l}\text { 1. Trois frères se querel- } \\
\text { lent; }\end{array}$ \\
\hline $\begin{array}{l}\text { 2a. ils sont mêlés à la } \\
\text { chasse au sanglier } \\
\text { 2b. nommé Trwyth }\end{array}$ & & $\begin{array}{l}\text { 2a. Corc est assailli comme } \\
\text { dans une chasse au } \\
\text { sanglier } \\
\text { 2b. à laquelle est mêlé celui } \\
\text { qui est aussi appelé Triath, }\end{array}$ \\
\hline $\begin{array}{l}\text { 3. dans laquelle Dillus joue } \\
\text { un rôle }\end{array}$ & & 3. c'est-à-dire Díl; \\
\hline $\begin{array}{l}\text { 4. un géant veut se faire un } \\
\text { manteau de leurs barbes }\end{array}$ & $\begin{array}{l}\text { 4. Dillus le Barbu est rasé } \\
\text { et tué... }\end{array}$ & \\
\hline $\begin{array}{l}\text { 5a. suite à un inceste } \\
\text { 5b. Peibio condamne sa fille } \\
\text { 5c. et leur fils nouveau-né } \\
\text { 5d. à être brûlés, } \\
\text { 5e. à être jetés à la mer } \\
\text { 5f. dans un sac } \\
\text { 5g. l'enfançon est sauvé } \\
\text { des eaux } \\
\text { 5h. et apporte la santé au roi }\end{array}$ & & $\begin{array}{l}5 \text { a à la suite d'un inceste, } \\
5 \text { b le roi condamne sa fille } \\
5 \text { c et ses fils nouveaux-nés, } \\
5 \text { d le premier à être brûlé } \\
\text { 5e et jeté à la mer; le second } \\
\text { à être emmené outremer } \\
5 \text { f d'où il revient sauvé; }\end{array}$ \\
\hline \multicolumn{3}{|l|}{$\begin{array}{l}\text { 6. Nynnio et Peibio sont } \\
\text { métamorphosés en } \\
\text { bovidés; }\end{array}$} \\
\hline $\begin{array}{l}\text { 7a. le Llyn Lliwan, source } \\
\text { cosmique } \\
\text { 7b. déborde et menace } \\
\text { d'inonder le pays; } \\
\text { 7c. Nynnio et Peibio } \\
\text { arrêtent l'éruption du lac; }\end{array}$ & $\begin{array}{l}\text { 7a ... au Plynlimon (centre } \\
\text { du monde gallois); } \\
\text { 7b. Peibio bave sans arrêt } \\
\text { 7c. mais est guéri. }\end{array}$ & \\
\hline $\begin{array}{l}\text { 8. Peibio perd un œil dans } \\
\text { ce genre d'aventure. }\end{array}$ & & 8. Díl a perdu un œil. \\
\hline
\end{tabular}

195. F. M. Gambari, "Il dio-toro sulle cime delle Alpi occidentale : indice di continuità nella tradizione culturale dalla Preistoria all'età romana ", Bulletin d'Etudes Préhistoriques et Archéologiques Alpines, XII, 2001, p. 10 n. 7 rapproche de cet affrontement final sous la forme de deux taureaux la coutume du " combat des reines " qui oppose encore aujourd'hui les deux plus belles vaches laitières de l'année dans le folklore valdotain... 


\section{RÉSUMÉ}

Diverses traditions galloises mettent en scène deux rois légendaires métamorphosés en bœufs et attachés d'une part à un formatage originel du pays (en tant que microcosme), d'autre part à un risque d'ennoiement eschatologique du même pays. Remarquablement, le même thème se retrouve dans les autres traditions celtes, jusqu'à la fameuse légende armoricaine de la submersion d'Is, de sorte qu'il apparaît vraisemblable qu'il y a des échos de très anciennes croyances, sans doute préchrétiennes.

\section{ABSTRACT}

Several Welsh traditions tell about two legendary kings transformed into oxen and associated with a (microcosmic) primal shaping of the landscape, and with an eventual eschatological flood. As a matter of fact, similar tales are known into other Celtic lores, as the notorious Breton legend about the sinking of Is, and they seem to point at very ancient, even prechristian beliefs. 